\title{
Autorías, colaboración y patrones de citación de las revistas biomédicas editadas en España incluidas en el Journal Citation Reports (2003-2007)
}

\author{
Gregorio González-Alcaide*
}

Resumen: Se realiza un análisis bibliométrico de las revistas biomédicas editadas en España recogidas en el Journal Citation Reports entre 2003 y 2007. Se han analizado 12.335 trabajos, un 86,4\% publicados en once revistas editadas en español y un 13,59\% en cinco revistas editadas en inglés. La gran mayoría de revistas editadas en español son publicaciones oficiales de sociedades médicas, constituyendo un importante canal de comunicación científica para sus miembros. Estas revistas aglutinan un importante número de grupos de investigación $(n=525)$, el 85,8\% de los trabajos de las mismas han sido firmados por centros asistenciales hospitalarios, con un predominio de la colaboración nacional y regional. En estas publicaciones, los trabajos realizados por los grupos de investigación identificados, aquellos con índices de colaboración más elevados y los trabajos en los que ha participado alguna mujer, presentan mayores tasas de citación. Por otra parte, las revistas editadas en inglés recogen fundamentalmente investigaciones básicas y solamente publican artículos y revisiones. Estas revistas presentan una productividad más reducida, pero una destacada participación internacional y un elevado grado de citación. El 87,62\% de estos trabajos han sido publicados en el ámbito académico y la colaboración internacional oscila entre el 16 y el $22 \%$ de las colaboraciones.

Palabras clave: Biomedicina, revistas científicas, bibliometría, autorías, colaboración científica, indicadores de citación, España.

\section{Authorship, collaboration and citation patterns of biomedical journals published in Spain and included in Journal Citation Reports (2003-2007)}

Abstract: A bibliometric analysis of Spanish biomedical journals indexed in the Journal Citation Reports database between 2003 and 2007 was carried out. 12.335 papers were analysed, 86.4\% published in eleven Spanish language journals and $13.59 \%$ published in five English language journals. The great majority of Spanish language journals are official publications of Spanish medical societies which represent for their members an important channel for scholarly communication. The Spanish language journals revealed a notable number of Spanish research groups $(n=525)$, 85.8\% of published

* Departamento de Historia de la Ciencia y Documentación. Instituto de Historia de la Medicina y de la Ciencia López Piñero, Universitat de València-Consejo Superior de Investigaciones Científicas, Valencia, España. Correo-e: gregorio.gonzalez@uv.es.

Recibido: 31-8-09; 2. ${ }^{\text {a }}$ versiñon: 25-10-09; aceptado: 5-11-09. 
papers came from health care institutions, and the prevalent type of collaboration was domestic and regional. Furthermore, the study reveals that higher levels of citation are associated with papers published by the aforementioned research groups, those having a greater degree of collaboration, and those that had at least one woman participating. On the other hand, English language journals are focused on basic research and only publish articles or reviews. Their productivity rate is lower, but they have a higher level of international participation and outstanding citation rates. $87.62 \%$ of papers from these journals were published by researchers from academic institutions. Collaboration on the international level ranges between $16 \%$ and $22 \%$ of the total.

Keywords: Biomedicine, scholarly journals, bibliometrics, authorship, scientific collaboration, citation indicators, Spain.

\section{Introducción}

La investigación biomédica trata de incrementar el conocimiento de los mecanismos de funcionamiento del ser humano para aplicarlos en la mejora de la salud de los ciudadanos. En la investigación biomédica es posible diferenciar dos grandes áreas de conocimiento: las Ciencias de la Vida y la Medicina Clínica. Dentro de las Ciencias de la Vida se encuadran toda una serie de disciplinas que, a partir de los principios de las ciencias naturales, estudian las propiedades y características de los seres humanos, tratando de profundizar en el conocimiento de los mecanismos moleculares, bioquímicos y celulares que determinan su funcionamiento; mientras que la Medicina Clínica aglutina todas aquellas disciplinas orientadas a la búsqueda, estudio e interpretación de las manifestaciones patológicas o a la obtención de datos de los pacientes para aplicarlos al diagnóstico, tratamiento y prevención de las enfermedades (González Alcaide y otros, 2009b).

Son numerosos los factores que justifican la importancia de la investigación biomédica, que constituye uno de los principales ámbitos objeto de la atención por parte de las políticas científicas y absorbe una parte importante de los recursos destinados a la investigación. Entre ellos, que la salud constituye el bien más preciado de las personas, y por tanto, es un bien de interés público que debe ser objeto de atención preferente; que la sociedad demanda cada vez más una atención sanitaria individualizada, eficiente y de calidad; que el surgimiento de nuevas enfermedades y su rápida propagación o la mayor incidencia de patologías relacionadas con los nuevos estilos de vida, la movilidad o el envejecimiento poblacional, exigen de una creciente atención y actualización de los conocimientos; o que determinados sectores, como el farmacéutico, el relacionado con los instrumentos biomédicos y los dispositivos sanitarios y también el sector biotecnológico, tienen un destacado peso en la economía de los países desarrollados, siendo sectores que se nutren en gran medida de la transferencia de los resultados generados por la investigación biomédica (Dones Tacero y otros, 2008; Pérez Sedeño y Miranda Suárez, 2008). 
En la actualidad asistimos a un importante proceso de internacionalización de la Ciencia española, con un destacado salto cuantitativo en el número de revistas científicas biomédicas editadas en España recogidas como parte de la cobertura de las bases de datos Thomson Reuters (antes Thomson-ISI), considerada el principal referente a nivel evaluativo por parte de los organismos gestores de las políticas científicas (Aleixandre Benavent, 2009; González Alcaide y otros, 2008), por lo que el análisis de las características bibliométricas de los trabajos publicados por las revistas que han formado parte de la cobertura de esta base de datos a lo largo de los últimos años puede resultar de interés para diferentes colectivos implicados en el proceso de generación, difusión y evaluación de las investigaciones científicas, como investigadores en Ciencias de la Salud, editores de revistas científicas y gestores de la investigación. El objetivo del presente estudio es analizar las características de las autorías y de la colaboración de la literatura científica difundida en las revistas biomédicas editadas en España que formaban parte de la cobertura del Journal Citation Reports (JCR) a lo largo del período 2003-2007.

\section{Metodología}

Para la realización del estudio se han identificado las revistas biomédicas editadas en España recogidas en el JCR entre los años 2003 y 2007. A continuación se han seleccionado todos los artículos, cartas, editoriales y revisiones de estas publicaciones en la base de datos Science Citation Index-Expanded (SCI-Expanded), se ha descargado la información bibliográfica y se ha confeccionado una base de datos relacional para facilitar el tratamiento y el análisis de los datos. Se ha efectuado una cuidadosa supervisión manual de la información bibliográfica para homogeneizar la información de las autorías, comparando las adscripciones institucionales asociadas a las diferentes variantes de firmas susceptibles de corresponder a un mismo autor, procediendo a su homogeneización en caso necesario, adoptando la forma más completa de la firma, por ejemplo, la que recoge los dos apellidos o el nombre de pila del autor desarrollado.

Se han calculado diferentes indicadores bibliométricos de productividad, colaboración e impacto científico para cada una de las revistas: número absoluto de documentos por tipología documental y año de publicación, media de trabajos por ejemplar, número de firmas, índice de firmas por trabajo o índice de colaboración, índice de transitoriedad, número absoluto de citas recibidas, media de citas por trabajo y porcentaje de trabajos no citados. Para el cálculo de los indicadores se ha optado por un recuento holístico completo, presentándose diferentes estadísticos (desviación estándar, rango e intervalo de confianza con un nivel del significación del 95\%) con el objeto de valorar el grado de dispersión de los datos respecto a las medias aritméticas. Todos estos indicadores se han calculado considerando todas las tipologías documentales y únicamente a partir de los artículos originales, por ser la tipología documental donde se recogen de 
forma preferente los principales resultados de las investigaciones científicas. Las citas y los indicadores de citación se han determinado a partir del número total de citas recibidas por los trabajos analizados recogidas en la base de datos SCIExpanded, siendo la fecha de realización de las búsquedas el 1 de febrero de 2009. Se recoge, asimismo, el porcentaje que representan los trabajos españoles publicados por cada una de las revistas en relación con el total de trabajos en los que han participado instituciones españolas publicados en el conjunto de revistas de las áreas a las que se adscriben, para lo que se han realizado diferentes búsquedas documentales que han combinado el listado de revistas de cada una de las disciplinas recogidas en el JCR con el identificador geográfico "Spain" en el campo afiliación institucional, teniendo presentes las limitaciones de tipología documental y años correspondientes a la cobertura del estudio.

Se han identificado los grupos de investigación activos que han contribuido de forma habitual con sus trabajos en cada una de las publicaciones a lo largo del período estudiado. Para ello, se han determinado y cuantificado todas las coautorías, información sobre la que se ha ejecutado un algoritmo que ha evaluado el umbral o intensidad de colaboración y el número mínimo de autores vinculados entre sí necesarios para considerar la existencia de un grupo de investigación, valores que se han fijado en la existencia de dos o más autores vinculados entre sí con tres o más relaciones de coautoría. Se ha determinado en cada caso, el porcentaje de autores integrados en los grupos y el número de investigadores del grupo más numeroso. Para la construcción de la red de autores que se presenta y para la identificación de los grupos, se ha utilizado el programa de análisis y visualización de redes Pajek (http://pajek.imfm.si/doku.php).

También se analiza el grado de participación de hombres y mujeres en las publicaciones por umbrales de productividad, la participación por sector institucional en los trabajos (centros asistenciales hospitalarios, universidades, centros de investigación, administración pública, organizaciones sin ánimo de lucro y empresas) y la colaboración institucional, para lo que se han identificado todos los tipos de colaboración presentes en cada uno de los documentos: colaboración intrainstitucional (documentos firmados conjuntamente por diferentes departamentos, servicios o unidades de una misma institución); colaboración interinstitucional (trabajos realizados en colaboración entre dos o más instituciones, diferenciando en este caso en los documentos firmados por instituciones españolas las colaboraciones entre instituciones de la misma o de diferentes Comunidades Autónomas); y colaboración internacional (trabajos firmados en colaboración entre instituciones de diferentes países). Finalmente, se analiza la evolución de los factores de impacto en el JCR de las publicaciones analizadas a lo largo del período objeto de cobertura por parte del estudio. Para la asignación del sexo de los autores, se ha desarrollado su nombre de pila cuando no se hacía constar en la base datos. Para ello, se ha consultado la base de datos de médicos colegiados de la Organización Médica Colegial de España; bases de datos y directorios de profesionales de diferentes áreas de las Ciencias de la Salud, como Medibooks, colegios médicos, Ministerio de Sanidad y Consumo, Consejerías de Salud o de 
las propias instituciones de adscripción de los autores; o se ha accedido al texto completo de los trabajos en el caso de las revistas que desarrollan los nombres de pila de los autores.

\section{Resultados}

Las revistas analizadas han publicado 12.335 trabajos, 10.658 trabajos publicados en las 11 revistas editadas en español $(86,4 \%)$ y 1.677 trabajos en las cinco revistas editadas en inglés (13,59\%). Medicina Clínica es la revista editada en español más productiva $(n=2.680)$, seguida por Revista de Neurología $(n=1.846)$ y Revista Española de Cardiología $(n=1.073)$. Otras seis publicaciones se sitúan por encima de los 500 trabajos, y únicamente dos revistas presentan un número inferior de trabajos (Actas Españolas de Psiquiatría y Neurocirugía). En relación con las revistas editadas en inglés, la más productiva es Histology and Histopathology ( $n=688$ ), seguida por International Journal of Developmental Biology $(n=484)$, situándose las tres revistas restantes por debajo de los 200 trabajos (tabla I).

Nefrología es la revista editada en español que presenta la media más elevada de artículos por ejemplar (12,6) y Revista Española de Enfermedades Digestivas la publicación con la media más baja $(6,51)$. Cabe destacar el elevado promedio de cartas por ejemplar recogidas en Medicina Clinica (10,51), presentando todas ellas un número variable de editoriales y revisiones. La distribución de los trabajos en las revistas editadas en inglés se presenta polarizada entre artículos y revisiones, no publicando ninguna de estas revistas cartas y solamente algunas de ellas un número reducido de editoriales (tabla I).

La evolución diacrónica del número de trabajos publicados por año presenta una gran estabilidad, tanto en las revistas editadas en español como en las revistas editadas en inglés. Medicina Clínica se sitúa por encima de los 500 trabajos/ año, situándose la práctica totalidad de revistas editadas en español en la franja comprendida entre los 97-232 trabajos/año, con la salvedad de Actas Españolas de Psiquiatría y Neurocirugía, que presentan una productividad menor, situada entre 58-63 trabajos/año. Todas las revistas editadas en inglés se sitúan por debajo de los 150 trabajos/año, con tres de ellas con una productividad anual mucho más reducida, comprendida entre los 23-42 trabajos/año (Aids Reviews, International Microbiology y Journal of Physiology and Biochemistry).

Las revistas editadas en español, que pertenecen todas ellas a disciplinas del área de la Medicina Clínica, canalizan una parte importante de la producción científica española en sus respectivas disciplinas según la clasificación de revistas del JCR. Así, Medicina Clínica ha difundido el 59,16\% y Revista Clinica Española el 22,06\% de los trabajos firmados por instituciones españolas del área de la Medicina General e Interna; Revista de Neurología reúne el 31,08\% y Neurología el 12,15\% de los trabajos españoles de la Neurología Clínica; Archivos de Bronconeumología aglutina el 41,67\% de los trabajos firmados por instituciones espa- 


\begin{tabular}{|c|c|c|c|c|c|c|c|c|c|c|c|c|c|c|c|c|c|c|c|}
\hline \multirow{6}{*}{ 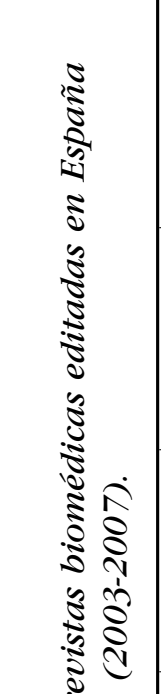 } & \multirow{2}{*}{ हुँ } & 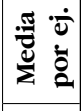 & $\begin{array}{l}n \\
0 \\
0 \\
0\end{array}$ & $\begin{array}{l}n \\
\infty \\
= \\
=\end{array}$ & है & 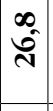 & $\begin{array}{l}\tilde{2} \\
\hat{\tilde{N}} \\
\hat{\sim}\end{array}$ & $\begin{array}{l}\hat{n} \\
\hat{0} \\
-1\end{array}$ & 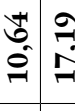 & 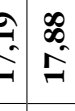 & $\stackrel{\sim}{\sim}$ & $\begin{array}{l}\infty \\
2 \\
n \\
n\end{array}$ & \begin{tabular}{c}
$n$ \\
\multirow{2}{*}{} \\
$\mathscr{f}^{\prime}$
\end{tabular} & $\begin{array}{l}n^{2} \\
\infty\end{array}$ & $\begin{array}{c}\hat{n} \\
\stackrel{\sim}{*}\end{array}$ & $\widehat{\tilde{\mathfrak{c}}}$ & $\frac{n}{\alpha}$ & $r$ & $\stackrel{n}{n}$ \\
\hline & & 莫 & $\begin{array}{l}\tilde{B} \\
\dot{n}\end{array}$ & $\bar{r}$ & $\overbrace{\infty}^{n}$ & 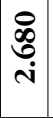 & 웅 & $\vec{n}$ & బิ & $\hat{~}$ & $\widetilde{\kappa}$ & 总 & $\left(\begin{array}{l}\infty \\
0 \\
6 \\
0 \\
0\end{array}\right.$ & $\stackrel{0}{\circ}$ & $\begin{array}{l}\infty \\
\infty \\
0\end{array}$ & $\stackrel{\infty}{+\infty}$ & 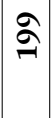 & $\stackrel{8}{+}$ & $\underset{n}{n}$ \\
\hline & : & 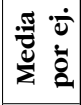 & 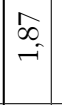 & $\mid \begin{array}{c}1 \\
\infty \\
0 \\
0\end{array}$ & $\stackrel{\infty}{2}$ & 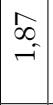 & 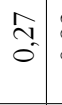 & $\approx$ & 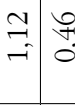 & \begin{tabular}{l|l}
0 & $\infty$ \\
0 & 0 \\
0 & 0
\end{tabular} & $\begin{array}{l}\text { in } \\
\text { ç } \\
0\end{array}$ & $\begin{array}{l}\stackrel{0}{2} \\
i \\
i\end{array}$ & $\begin{array}{l}\infty \\
\mathfrak{N} \\
\sim \\
\sim\end{array}$ & $\begin{array}{l}n \\
6 \\
2\end{array}$ & $\begin{array}{l} \pm \\
\vec{a}\end{array}$ & $\stackrel{\hat{~}}{\approx}$ & $\mid$ & $\begin{array}{l}n \\
\infty \\
0 \\
0\end{array}$ & $\begin{array}{l}n \\
n \\
n\end{array}$ \\
\hline & 苛 & 完 & 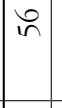 & $\vec{n}$ & భे & $\infty$ & $\infty$ & $\stackrel{\sim}{\sim}$ & $\stackrel{N}{\sim}$ & $\widehat{v} \stackrel{\infty}{+}$ & $\stackrel{i n}{\sim}$ & $\begin{array}{l}\hat{D} \\
\text { r }\end{array}$ & $\tilde{\omega}$ & $m$ & $\begin{array}{l}\stackrel{0}{a} \\
\stackrel{n}{v}\end{array}$ & $\vec{r}$ & $\hat{n}$ & 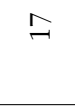 & $\stackrel{+}{*}$ \\
\hline & $\frac{\ddot{\theta}}{3}$ & 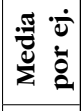 & $\begin{array}{l}n \\
- \\
0\end{array}$ & $\stackrel{n}{\approx}$ & $\begin{array}{r}\tilde{n} \\
n^{2}\end{array}$ & $\left|\begin{array}{l}f \\
i f \\
i n\end{array}\right|$ & $N$ & $\begin{array}{l}\approx \\
\tilde{v}\end{array}$ & 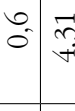 & $\begin{array}{l}\vec{n} \\
\vec{f} \\
\tilde{\sigma}\end{array}$ & $\begin{array}{l}\text { ñ } \\
\text { cis }\end{array}$ & $\tilde{\alpha}$ & $\begin{array}{c}\infty \\
\infty \\
i\end{array}$ & $\mid$ & 0 & $\frac{1}{0}$ & $\rightarrow$ & 0 & $\begin{array}{c}\infty \\
\infty \\
0 \\
0\end{array}$ \\
\hline & 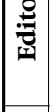 & 竞 & 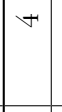 & $\infty$ & $\stackrel{0}{0}$ & $\begin{array}{l}i f \\
i n \\
i n\end{array}$ & 8 & $R$ & กิ & $\vec{\imath}$ & $\stackrel{n}{n}$ & $\exists$ & $\begin{array}{c}N \\
\tilde{\infty} \\
\infty \\
-\end{array}$ & in & 0 & ৯ે & ㄱ. & - & $\stackrel{1}{\hat{\theta}}$ \\
\hline 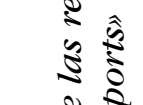 & ב & 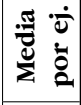 & $\mid \begin{array}{l}\mathscr{P} \\
0 \\
0\end{array}$ & $\begin{array}{l}n \\
i \\
i\end{array}$ & $\stackrel{\infty}{\stackrel{\infty}{\hbar}}$ & $\begin{array}{l}\vec{n} \\
\hat{n}\end{array}$ & $\begin{array}{l}\vec{f} \\
\text { in }\end{array}$ & $\overrightarrow{0}$ & 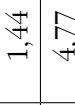 & 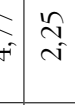 & $\stackrel{\infty}{\underset{f}{*}}$ & $\begin{array}{l}\infty \\
i n \\
i\end{array}$ & $\stackrel{f}{f}$ & 0 & 0 & 0 & 0 & 0 & 0 \\
\hline 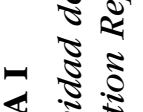 & ज्ञ & 莫 & $\because$ & $\stackrel{I}{\exists}$ & ते & $\mid \begin{array}{r}\vec{n} \\
\stackrel{0}{-i}\end{array}$ & $\stackrel{\vec{b}}{\overrightarrow{-}}$ & $n$ & N & $\begin{array}{c}v \\
v\end{array}$ & $\stackrel{\sqrt{n}}{\sqrt{n}}$ & $\stackrel{0}{m}$ & $\begin{array}{l}\hat{i} \\
i \\
i\end{array}$ & 0 & 0 & 0 & 0 & 0 & 0 \\
\hline 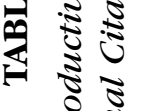 & $\mid \begin{array}{l}0 \\
\frac{0}{z} \\
\bar{z}\end{array}$ & 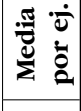 & $\begin{array}{l}0 \\
0 \\
1\end{array}$ & in & $\delta^{\prime}$ & $\begin{array}{l}a \\
\infty \\
\infty\end{array}$ & $\begin{array}{l}0 \\
\text { i } \\
-1\end{array}$ & 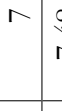 & 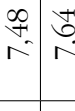 & $\begin{array}{ll}\tilde{N} \\
\tilde{0} \\
\tilde{n}\end{array}$ & $\sqrt{n}$ & $\vec{n}$ & $\begin{array}{l}\stackrel{+}{N} \\
\infty \\
\infty\end{array}$ & $\stackrel{\infty}{-}$ & $\begin{array}{l}\stackrel{7}{7} \\
\text { in }\end{array}$ & $\stackrel{0}{a}$ & $\vec{\pi}$ & $\overrightarrow{6}$ & $\left|\begin{array}{c}N \\
N \\
\infty\end{array}\right|$ \\
\hline 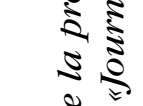 & 焉 & 豆 & 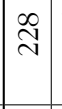 & 官 & $\sqrt{n}$ & $\hat{\infty}$ & $\begin{array}{l}\infty \\
n \\
n\end{array}$ & $\underset{\sim}{\stackrel{ }{v}}$ & 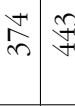 & $f=\vec{F}$ & $\overrightarrow{\grave{n}}$ & 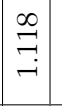 & $\begin{array}{l}\hat{n} \\
\hat{n} \\
\dot{n}\end{array}$ & in & $\underset{\sim}{\widetilde{F}}$ & $\stackrel{+}{\infty}$ & $\stackrel{\Im}{\beth}$ & $\underset{\exists}{ }$ & 号 \\
\hline 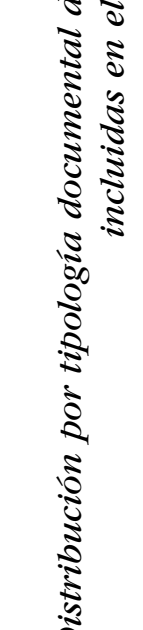 & & 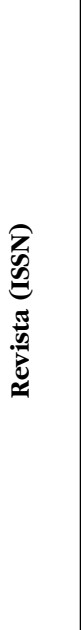 & 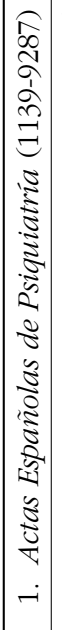 & 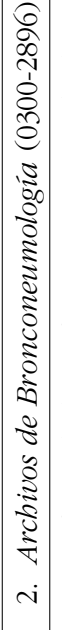 & 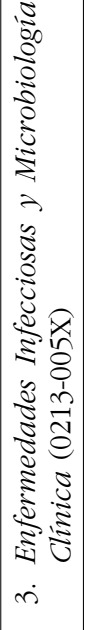 & 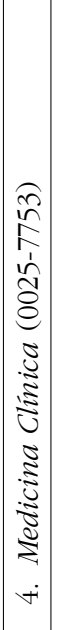 & 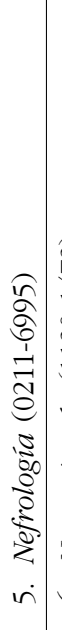 & 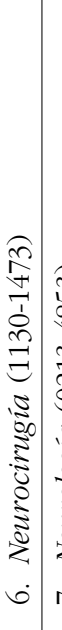 & 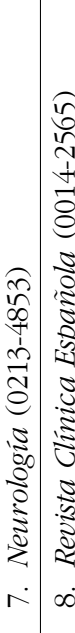 & 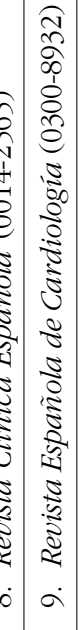 & $\begin{array}{ll} \\
0 \\
0 \\
0 \\
0 \\
0 \\
0 \\
0 \\
0 \\
0 \\
0 \\
0 \\
0 \\
0 \\
0 \\
0 \\
0 \\
0 \\
0 \\
0 \\
0 \\
0 \\
0 \\
0 \\
0 \\
0 \\
0 \\
0 \\
0 \\
0 \\
0 \\
0 \\
0 \\
0 \\
0 \\
0 \\
0\end{array}$ & 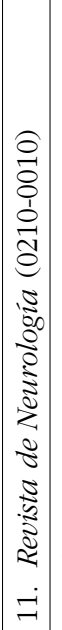 & : & 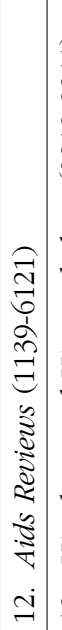 & 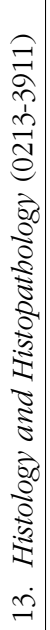 & 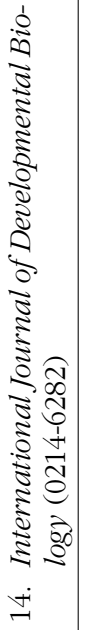 & 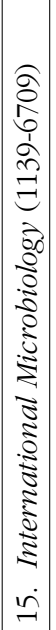 & 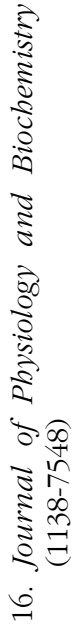 & \begin{tabular}{|c|}
$\bar{\pi}$ \\
0 \\
$=$
\end{tabular} \\
\hline & & & & & & & & & & & & & & & & & & & \\
\hline
\end{tabular}


ñolas en el área del Sistema respiratorio; Revista Española de Cardiología reúne el 36,6\% de los trabajos firmados por al menos una institución española en el área del Sistema Cardíaco y Cardiovascular; Revista Española de Enfermedades Digestivas recoge el 33,12\% de los trabajos de instituciones españolas del área de Gastroenterología y Hepatología; Nefrología el 31,56\% de los trabajos en el área de Urología y Nefrología; Enfermedades Infecciosas y Microbiología Clínica el 26,29\% de trabajos firmados por instituciones españolas en el área de las Enfermedades Infecciosas; Actas Españolas de Psiquiatría el 19,45\% de los trabajos españoles en el área de la Psiquiatría; y finalmente, Neurocirugía el 8,64\% de los trabajos firmados por instituciones españolas en el área de la Cirugía. En relación con estos aportes, las revistas editadas en español son las que reúnen los porcentajes más elevados de participación española (tabla II).

Ha de tenerse en cuenta que todas las revistas editadas en español están adscritas a diferentes disciplinas del área de la Medicina Clínica de la clasificación temática de revistas del Journal Citation Reports, correspondiendo a estas áreas los aportes, si bien, tres de ellas están además multiasignadas a disciplinas del área de las Ciencias de la Vida: Actas Españolas de Psiquiatría (Neurociencias), Enfermedades Infecciosas y Microbiología Clinica (Microbiología) y Neurocirugía (Neurociencias). Todas las revistas editadas en inglés están clasificadas temáticamente como pertenecientes a disciplinas del área de las Ciencias de la Vida, y únicamente Aids Reviews está asignada a Enfermedades Infecciosas (Medicina Clínica). Asimismo, International Microbiology está también asignada a la categoría Biotecnología y Microbiología Aplicada, que no ha sido considerada para el análisis (Biomedicina) porque en el JCR aparece descrita como una disciplina orientada a la manipulación de organismos vivos para resolver problemas relacionados con la alimentación humana. En el caso de las revistas de las Ciencias de la Vida, se presentan los aportes que corresponderían al recuento de la totalidad de los trabajos en cada una de las áreas de las Ciencias de la Vida a las que están asignadas.

En el conjunto de trabajos han intervenido 28.043 autores diferentes, que son responsables de 52.903 firmas, con 406 grandes productores (autores con >9 trabajos) y 18.915 autores transitorios (autores que han participado en un único trabajo). En los 6.453 artículos originales se han identificado 20.387 autores, responsables de 33.718 firmas, con 140 grandes productores y 14.571 autores transitorios. La mayor parte de los grandes productores se han identificado en las revistas editadas en español $(n=388)$, que presentan en los artículos originales un índice de firmas por trabajo superior $(5,33 \pm 3,01)$ a las revistas editadas en inglés $(4,72 \pm 2,57)$ (tabla III).

Considerando únicamente los artículos originales, Revista Española de Cardiología es la revista editada en español que presenta el índice de firmas por trabajo más elevado $(7,11 \pm 4,15)$, seguida por Nefrología $(6,34 \pm 3,6)$, Archivos de Bronconeumología $(5,59 \pm 2,13)$, Medicina Clinica $(5,54 \pm 2,61)$, Revista Española de Enfermedades Digestivas $(5,54 \pm 2,85)$ y Enfermedades Infecciosas y Microbiología Clínica $(5,51 \pm 3,41)$. El resto de revistas se sitúan con valores com- 


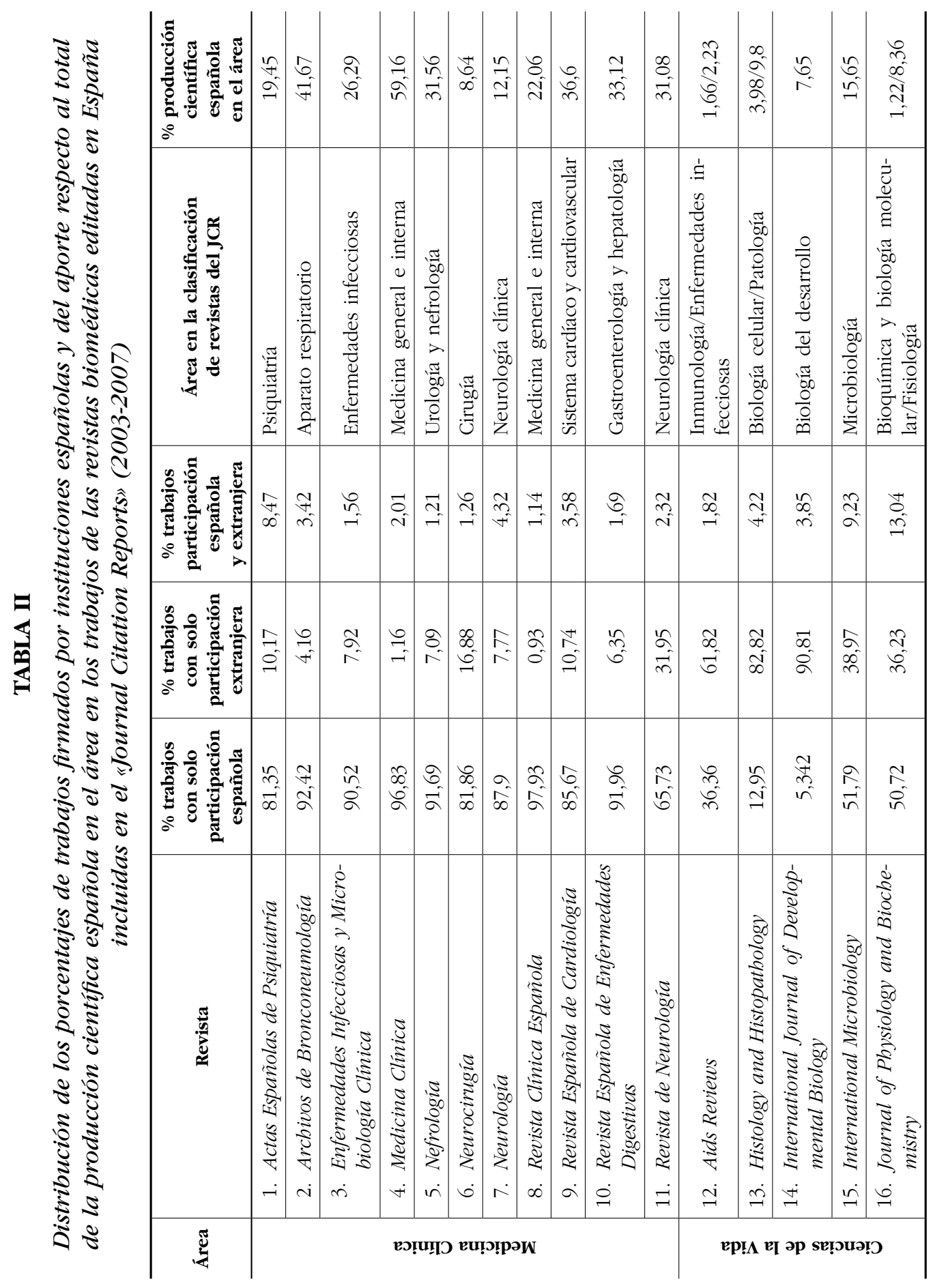


prendidos entre 4,38 y 4,96. Entre las revistas editadas en inglés, solamente Histology and Histopathology $(5,92 \pm 2,51)$ y Journal of Physiology and Biochemistry $(5,22 \pm 2,03)$ se sitúan con valores superiores a cinco, presentando el resto de revistas un menor grado de colaboración, con índices de firmas por trabajo situados entre 2,72 y 4,07. En relación con los índices de transitoriedad, tres revistas editadas en español se sitúan con valores por encima del 80\%: $R$ evista Clinica Española (85,95\%), Actas Españolas de Psiquiatría (82,27\%) y Medicina Clínica (81,19\%), situándose el resto de publicaciones con valores comprendidos entre el 66,33\% de Nefrologia y el 78,26\% de Enfermedades Infecciosas y Microbiología Clínica. Las revistas editadas en inglés presentan índices de transitoriedad mucho más elevados, que oscilan entre el 83,16\% de Journal of Physiology and Biochemistry y el 94,62\% de Aids Reviews (tabla III).

En relación con la participación por sectores institucionales, los centros asistenciales hospitalarios han participado en el 70,15\% de los artículos originales $(n=4.527)$, las universidades en el 38,48\% $(n=2.483)$, los centros de investigación en el 13,93\% $(n=899)$, la administración pública en el 5,18\% $(n=334)$, los centros asistenciales no hospitalarios en el 4,34\% $(n=280)$, las empresas en el 3,11\% $(n=201)$ y las organizaciones sin ánimo de lucro en el 3,1\% $(n=200)$. Considerando únicamente las revistas editadas en español, el peso de los centros asistenciales hospitalarios es aún mayor, ya que han participado en el 85,8\% $(n=4.334)$ de los artículos originales frente a una participación de únicamente el 17,74\% ( $n=195)$ en las revistas editadas en inglés, publicaciones en las que las universidades se sitúan en primer lugar, con una participación en el 87,62\% de los artículos $(n=963)$, teniendo también una destacada importancia en estas publicaciones los centros de investigación, con una participación en el 28,3\% de los trabajos $(n=311)$.

El algoritmo ejecutado sobre las coautorías identificadas (2 o más autores vinculados entre sí con $\geq 3$ trabajos en colaboración), ha permitido determinar la existencia en el conjunto de revistas analizadas de 608 grupos de investigación integrados por 3.033 autores, valores que se reducen sensiblemente a medida que se incrementa el umbral de colaboración necesario para considerar la existencia de un grupo de investigación. La mayor parte de los grupos han sido identificados en las revistas españolas $(n=543)$, presentando cada una de ellas un número variable de grupos, pudiéndose destacar Medicina Clínica, con 112 grupos; Revista de Neurología, con 99 grupos; Revista Española de Cardiología, con 54; Nefrología y Revista Española de Enfermedades Digestivas, con 46 grupos cada de una de ellas, respectivamente; y Archivos de Bronconeumología, con 41. Las revistas editadas en inglés reúnen un número reducido de grupos $(n=60)$, con la única excepción de Histology and Histopathology, en la que se han identificado 38 grupos (tabla IV).

Entre las revistas editadas en español ( $\geq 3$ trabajos en colaboración), Neurocirugía es la publicación que presenta un mayor porcentaje de autores que participan en la revista integrados en alguno de los grupos de investigación identificados $(76,41 \%$ de los autores que han publicado tres o más trabajos), seguida 
TABLA III

Características de las autorías e indicadores de colaboración de los trabajos publicados por las revistas biomédicas editadas en España incluidas en el «Journal Citation Reports»(2003-2007).

\begin{tabular}{|c|c|c|c|c|c|c|}
\hline \multirow[b]{2}{*}{ Área } & \multirow[b]{2}{*}{ Revista } & \multicolumn{5}{|c|}{ Todos los documentos } \\
\hline & & $\begin{array}{c}N^{\circ} \\
\text { firmas }\end{array}$ & $\begin{array}{c}\text { Índ. de firmas/ } \\
\text { trabajo } \pm \text { DS } \\
\text { (IC al } 95 \% \text { ) }\end{array}$ & $\begin{array}{l}\mathrm{N}^{\circ} \mathrm{de} \\
\text { autores } \\
\text { (rango) }\end{array}$ & $\begin{array}{l}\mathbf{N}^{\mathbf{0}} \\
\mathbf{G P}\end{array}$ & $\begin{array}{c}\text { Índ. de } \\
\text { transito- } \\
\text { riedad (\%) }\end{array}$ \\
\hline \multirow{12}{*}{ 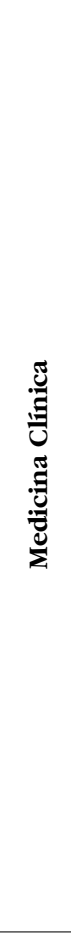 } & 1. Actas Españolas de Psiquiatría & 1.341 & $\begin{array}{l}4,45 \pm 2,34 \\
(4,18-4,71)\end{array}$ & $\begin{array}{r}970 \\
(1-15)\end{array}$ & 1 & 80,62 \\
\hline & 2. Archivos de Bronconeumología & 3.087 & $\begin{array}{l}4,34 \pm 2,53 \\
(4,15-4,52)\end{array}$ & $\begin{array}{r}1.810 \\
(1-22)\end{array}$ & 14 & 70,44 \\
\hline & $\begin{array}{l}\text { 3. Enfermedades Infecciosas y Micro- } \\
\text { biología Clinica }\end{array}$ & 3.612 & $\begin{array}{l}4,49 \pm 3,23 \\
(4,26-4,71)\end{array}$ & $\begin{array}{r}2.289 \\
(1-25)\end{array}$ & 10 & 72,21 \\
\hline & 4. Medicina Clínica & 10.078 & $\begin{array}{l}3,76 \pm 2,27 \\
(3,67-3,84)\end{array}$ & $\begin{array}{r}6.650 \\
(1-20)\end{array}$ & 29 & 74,31 \\
\hline & 5. Nefrología & 3.171 & $\begin{array}{l}5,2 \pm 3,52 \\
(4,92-5,48)\end{array}$ & $\begin{array}{r}1.622 \\
(1-33) \\
\end{array}$ & 26 & 64,55 \\
\hline & 6. Neurocirugia & 1.294 & $\begin{array}{l}4,16 \pm 2,55 \\
(3,88-4,44)\end{array}$ & $\begin{array}{r}730 \\
(1-14) \\
\end{array}$ & 12 & 71,23 \\
\hline & 7. Neurología & 2.019 & $\begin{array}{l}3,79 \pm 2,59 \\
(3,57-4,01)\end{array}$ & $\begin{array}{r}1.335 \\
(1-21)\end{array}$ & 7 & 75,73 \\
\hline & 8. Revista Clínica Española & 4.185 & $\begin{array}{c}4,2 \pm 2,3 \\
(4,06-4,34)\end{array}$ & $\begin{array}{r}2.992 \\
(1-21) \\
\end{array}$ & 8 & 79,11 \\
\hline & 9. Revista Española de Cardiología & 5.731 & $\begin{array}{c}5,34 \pm 4,03 \\
(5,1-5,58)\end{array}$ & $\begin{array}{r}3.068 \\
(1-60)\end{array}$ & 44 & 67,73 \\
\hline & $\begin{array}{l}\text { 10. Revista Española de Enfermedades } \\
\text { Digestivas }\end{array}$ & 3.954 & $\begin{array}{l}4,99 \pm 2,78 \\
(4,8-5,18)\end{array}$ & $\begin{array}{r}2.280 \\
(1-18) \\
\end{array}$ & 19 & 68,81 \\
\hline & 11. Revista de Neurología & 7.537 & $\begin{array}{l}4,08 \pm 2,54 \\
(3,96-4,19) \\
\end{array}$ & $\begin{array}{r}4.480 \\
(1-24) \\
\end{array}$ & 41 & 70,89 \\
\hline & Total $^{1}$ & 46.009 & $\begin{array}{l}4,32 \pm 2,83 \\
(4,27-4,37) \\
\end{array}$ & $\begin{array}{l}22.919 \\
(1-60)\end{array}$ & 388 & 64,01 \\
\hline \multirow{6}{*}{ 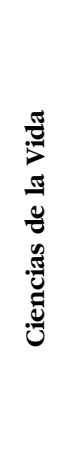 } & 12. Aids Reviews & 392 & $\begin{array}{l}2,36 \pm 2,16 \\
(2,03-2,69)\end{array}$ & $\begin{array}{r}304 \\
(1-20) \\
\end{array}$ & 1 & 85,2 \\
\hline & 13. Histology and Histopathology & 3.472 & $\begin{array}{l}5,05 \pm 2,61 \\
(4,85-5,24)\end{array}$ & $\begin{array}{r}2.643 \\
(1-20)\end{array}$ & 7 & 82,44 \\
\hline & $\begin{array}{l}\text { 14. International Journal of Develop- } \\
\text { mental Biology }\end{array}$ & 1.620 & $\begin{array}{l}3,35 \pm 2,17 \\
(3,16-3,54) \\
\end{array}$ & $\begin{array}{c}1.404 \\
(1-14)\end{array}$ & 1 & 89,39 \\
\hline & 15. International Microbiology & 694 & $\begin{array}{l}3,49 \pm 2,47 \\
(3,15-3,83)\end{array}$ & $\begin{array}{r}556 \\
(1-14) \\
\end{array}$ & 1 & 83,99 \\
\hline & $\begin{array}{l}\text { 16. Journal of Physiology and Bioche- } \\
\text { mistry }\end{array}$ & 716 & $\begin{array}{l}5,11 \pm 2,02 \\
(4,77-5,44) \\
\end{array}$ & $\begin{array}{r}524 \\
(1-11) \\
\end{array}$ & 2 & 83,59 \\
\hline & Total $^{1}$ & 6.894 & $\begin{array}{l}4,11 \pm 2,58 \\
(3,99-4,23)\end{array}$ & $\begin{array}{c}5.408 \\
(1-20)\end{array}$ & 12 & 84,34 \\
\hline
\end{tabular}

DS: Desviación estándar; IC: Intervalo de confianza; GP: grandes productores (autores con > 9 trabajos). (1) El número total de autores no se corresponde con la suma de los autores por revistas por el hecho de que los mismos autores intervienen en diferentes revistas. 


\section{TABLA III (continuación)}

\begin{tabular}{|c|c|c|c|c|c|c|}
\hline \multirow[b]{2}{*}{ Área } & \multirow[b]{2}{*}{ Revista } & \multicolumn{5}{|c|}{ Artículos originales } \\
\hline & & $\begin{array}{c}N^{\circ} \\
\text { firmas }\end{array}$ & $\begin{array}{c}\text { Índ. de firmas/ } \\
\text { trabajo } \pm \text { DS } \\
\text { (IC al 95\%) }\end{array}$ & $\begin{array}{l}\mathrm{N}^{\circ} \mathrm{de} \\
\text { autores } \\
\text { (rango) }\end{array}$ & $\begin{array}{l}\mathbf{N}^{\mathbf{o}} \\
\mathbf{G P}\end{array}$ & $\begin{array}{c}\text { Índ. de } \\
\text { transito- } \\
\text { riedad (\%) }\end{array}$ \\
\hline \multirow{12}{*}{ 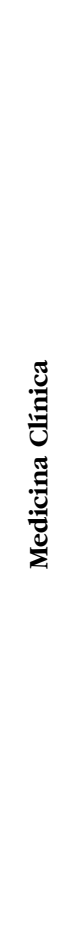 } & 1. Actas Españolas de Psiquiatría & 1.102 & $\begin{array}{l}4,83 \pm 2,35 \\
(4,52-5,13)\end{array}$ & $\begin{array}{r}818 \\
(1-15)\end{array}$ & 1 & 82,27 \\
\hline & 2. Archivos de Bronconeumología & 2.438 & $\begin{array}{l}5,59 \pm 2,13 \\
(5,39-5,79)\end{array}$ & $\begin{array}{c}1.536 \\
(1-18)\end{array}$ & 6 & 73,11 \\
\hline & $\begin{array}{l}\text { 3. Enfermedades Infecciosas y Micro- } \\
\text { biología Clinica }\end{array}$ & 1.933 & $\begin{array}{l}5,51 \pm 3,41 \\
(5,15-5,87)\end{array}$ & $\begin{array}{r}1.403 \\
(1-25)\end{array}$ & 0 & 78,26 \\
\hline & 4. Medicina Clinica & 4.969 & $\begin{array}{l}5,54 \pm 2,61 \\
(5,37-5,71) \\
\end{array}$ & $\begin{array}{r}3.758 \\
(1-20) \\
\end{array}$ & 3 & 81,19 \\
\hline & 5. Nefrología & 2.396 & $\begin{array}{l}6,34 \pm 3,6 \\
(5,98-6,7)\end{array}$ & $\begin{array}{r}1.372 \\
(1-31)\end{array}$ & 6 & 66,33 \\
\hline & 6. Neurocirugía & 1.041 & $\begin{array}{l}4,96 \pm 2,23 \\
(4,66-5,26)\end{array}$ & $\begin{array}{r}653 \\
(1-12)\end{array}$ & 3 & 71,97 \\
\hline & 7. Neurologia & 1.637 & $\begin{array}{l}4,38 \pm 2,65 \\
(4,11-4,65)\end{array}$ & $\begin{array}{r}1.157 \\
(1-21) \\
\end{array}$ & 2 & 78,56 \\
\hline & 8. Revista Clinica Española & 2.054 & $\begin{array}{l}4,64 \pm 2,8 \\
(4,38-4,9)\end{array}$ & $\begin{array}{r}1.659 \\
(1-21)\end{array}$ & 1 & 85,95 \\
\hline & 9. Revista Española de Cardiología & 3.633 & $\begin{array}{l}7,11 \pm 4,15 \\
(6,75-7,47)\end{array}$ & $\begin{array}{r}2.157 \\
(1-60)\end{array}$ & 19 & 72,18 \\
\hline & $\begin{array}{l}\text { 10. Revista Española de Enfermedades } \\
\text { Digestivas }\end{array}$ & 2.166 & $\begin{array}{l}5,54 \pm 2,85 \\
(5,25-5,82)\end{array}$ & $\begin{array}{r}1.437 \\
(1-18) \\
\end{array}$ & 6 & 74,25 \\
\hline & 11. Revista de Neurología & 5.085 & $\begin{array}{c}4,55 \pm 2,64 \\
(4,39-4,7)\end{array}$ & $\begin{array}{r}3.358 \\
(1-24)\end{array}$ & 16 & 74,81 \\
\hline & Total $^{1}$ & 28.454 & $\begin{array}{l}5,33 \pm 3,01 \\
(5,25-5,41)\end{array}$ & $\begin{array}{l}16.291 \\
(1-60)\end{array}$ & 130 & 68,36 \\
\hline \multirow{6}{*}{ 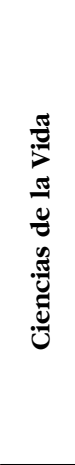 } & 12. Aids Reviews & 98 & $\begin{array}{c}2,72 \pm 1,58 \\
(2,2-3,24)\end{array}$ & $\begin{array}{c}93 \\
(1-8)\end{array}$ & 0 & 94,62 \\
\hline & 13. Histology and Histopathology & 2.557 & $\begin{array}{l}5,92 \pm 2,51 \\
(5,68-6,16)\end{array}$ & $\begin{array}{r}2.019 \\
(1-20)\end{array}$ & 5 & 83,95 \\
\hline & $\begin{array}{l}\text { 14. International Journal of Develop- } \\
\text { mental Biology }\end{array}$ & 1.394 & $\begin{array}{l}3,63 \pm 2,2 \\
(3,41-3,85)\end{array}$ & $\begin{array}{r}1.227 \\
(1-14) \\
\end{array}$ & 1 & 89,97 \\
\hline & 15. International Microbiology & 578 & $\begin{array}{l}4,07 \pm 2,53 \\
(3,65-4,49)\end{array}$ & $\begin{array}{r}473 \\
(1-14) \\
\end{array}$ & 0 & 84,78 \\
\hline & $\begin{array}{l}\text { 16. Journal of Physiology and Bioche- } \\
\text { mistry }\end{array}$ & 637 & $\begin{array}{l}5,22 \pm 2,03 \\
(4,86-5,58) \\
\end{array}$ & $\begin{array}{r}475 \\
(1-11) \\
\end{array}$ & 2 & 83,16 \\
\hline & Total $^{1}$ & 5.264 & $\begin{array}{l}4,72 \pm 2,57 \\
(4,57-4,87)\end{array}$ & $\begin{array}{r}4.274 \\
(1-20)\end{array}$ & 8 & 85,74 \\
\hline
\end{tabular}


TABLA IV

Grupos de investigación identificados a partir del análisis de coautorías de los trabajos publicados por las revistas biomédicas editadas en España incluidas en el «Journal Citation Reports»(2003-2007)

\begin{tabular}{|c|c|c|c|c|c|c|}
\hline Área & Revista & $\begin{array}{l}\text { Intensidad } \\
\text { de } \\
\text { colabora- } \\
\text { ción }\end{array}$ & $\begin{array}{l}\text { Número } \\
\text { de } \\
\text { grupos }\end{array}$ & $\begin{array}{c}\text { Número } \\
\text { de autores } \\
\text { en los } \\
\text { grupos }\end{array}$ & $\begin{array}{c}\% \\
\text { autores } \\
\text { integrados } \\
\text { en grupos }\end{array}$ & $\begin{array}{c}\mathbf{N}^{\mathbf{o}} \\
\text { miembros } \\
\text { grupo } \\
\text { mayor } \\
\text { tamaño }\end{array}$ \\
\hline \multirow{12}{*}{ 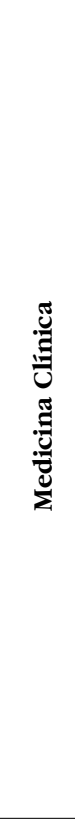 } & $\begin{array}{l}\text { 1. Actas Españolas de Psiquia- } \\
\text { tría }\end{array}$ & $\geq 3$ & 13 & 41 & 51,25 & 7 \\
\hline & $\begin{array}{l}\text { 2. Archivos de Bronconeumo- } \\
\text { logía }\end{array}$ & $\geq 3$ & 41 & 171 & 66,28 & 19 \\
\hline & $\begin{array}{l}\text { 3. Enfermedades Infecciosas y } \\
\text { Microbiología Clinica }\end{array}$ & $\geq 3$ & 36 & 138 & 52,47 & 47 \\
\hline & 4. Medicina Clinica & $\geq 3$ & 112 & 364 & 52 & 16 \\
\hline & 5. Nefrologia & $\geq 3$ & 46 & 231 & 71,07 & 23 \\
\hline & 6. Neurocirugía & $\geq 3$ & 15 & 81 & 76,41 & 15 \\
\hline & 7. Neurología & $\geq 3$ & 24 & 74 & 52,86 & 9 \\
\hline & 8. Revista Clínica Española & $\geq 3$ & 39 & 128 & 54,01 & 10 \\
\hline & $\begin{array}{l}\text { 9. Revista Española de Cardio- } \\
\text { logía }\end{array}$ & $\geq 3$ & 54 & 371 & 72,6 & 90 \\
\hline & $\begin{array}{l}\text { 10. Revista Española de Enfer- } \\
\text { medades Digestivas }\end{array}$ & $\geq 3$ & 46 & 252 & 73,68 & 27 \\
\hline & 11. Revista de Neurología & $\geq 3$ & 99 & 438 & 69,63 & 28 \\
\hline & Total $^{1}$ & $\geq 3$ & 543 & 2.796 & 62,54 & 287 \\
\hline \multirow{6}{*}{ 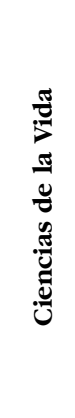 } & 12. Aids Reviews & $\geq 3$ & 2 & 4 & 26,67 & 2 \\
\hline & $\begin{array}{l}\text { 13. Histology and Histopatho- } \\
\log y\end{array}$ & $\geq 3$ & 38 & 141 & 82,45 & 13 \\
\hline & $\begin{array}{l}\text { 14. International Journal of De- } \\
\text { velopmental Biology }\end{array}$ & $\geq 3$ & 8 & 21 & 53,84 & 4 \\
\hline & 15. International Microbiology & $\geq 3$ & 4 & 12 & 54,54 & 6 \\
\hline & $\begin{array}{l}\text { 16. Journal of Physiology and } \\
\text { Biochemistry }\end{array}$ & $\geq 3$ & 8 & 37 & 90,24 & 10 \\
\hline & Total $^{1}$ & $\geq 3$ & 60 & 215 & 73,88 & 13 \\
\hline
\end{tabular}

(1) El número total de autores no se corresponde con la suma de los autores por revistas por el hecho de que los mismos autores intervienen en diferentes revistas. 
por Revista Española de Enfermedades Digestivas (73,68\%), Revista Española de Cardiología (72,5\%), Nefrología (71,08\%), Revista de Neurología (69,63\%) y Archivos de Bronconeumología (66,28\%); situándose el resto de publicaciones con valores por debajo del $60 \%$ de los autores que han publicado tres o más trabajos integrados en grupos. Cabe destacar asimismo que Revista Española de Cardiología es la publicación que presenta el grupo con mayor número de miembros vinculados entre sí, bien de forma directa o a través de intermediarios $(n=90)$. Ya a cierta distancia se sitúan Enfermedades Infecciosas y Microbiología Clinica $(n=47)$, Revista de Neurología $(n=28)$, Revista Española de Enfermedades Digestivas $(n=27)$ y Nefrología $(n=23)$ (tabla IV).

En la figura 1 se presenta la red con los principales autores ( $>5$ trabajos) vinculados a las publicaciones en las que han participado, pudiendo apreciarse que las revistas de Medicina General e Interna Medicina Clinica y Revista Clinica Española comparten algunos autores que participan indistintamente en ambas publicaciones con un número importante de trabajos, si bien, solamente la primera de ellas, comparte un núcleo de autores destacado con revistas de otras especialidades, como Revista Española de Cardiología, Enfermedades Infecciosas y Microbiología Clinica, Archivos de Bronconeumología, Revista de Neurología y de forma más puntual, con Revista Española de Enfermedades Digestivas y $\mathrm{Ne}$ frología. Cabe destacar la escasa relación interdisciplinar más allá de estos vínculos, escasa relación que también se constata entre las revistas clasificadas en las mismas áreas temáticas, como Revista de Neurología y Neurología (Neurología Clínica) o Actas Españolas de Psiquiatría y Neurocirugía (Neurociencias), cada una de las cuales presenta sus respectivos autores y grupos de investigadores, autores que salvo casos puntuales, no están interrelacionados ni entre sí ni con las dos publicaciones de manera simultánea. Finalmente, es importante advertir que cuatro de las cinco revistas editadas en inglés (International Journal of Developmental Biology, Journal od Physiology and Biochemistry, International Microbiology e Histology and Histopathology), se presentan de forma aislada del conjunto de la red, cada una de ellas con sus respectivos autores y grupos, integrándose únicamente Aids Reviews en el conjunto de la red de revistas editadas en español.

Se ha identificado el sexo del 74,89\% $(n=6.845)$ de los autores que han publicado más de un trabajo en el conjunto de las publicaciones analizadas, con independencia de la tipología documental en la que han participado, de los que 4.580 son hombres $(66,91 \%)$ y 2.265 mujeres (33,09\%); y del $96,04 \%$ de los autores que han publicado más de dos trabajos $(n=4.634)$, de los que 3.257 (70,28\%) son hombres y 1.377 (29,71\%) mujeres. La distribución porcentual de hombres y mujeres por niveles de productividad muestra una reducción progresiva en el número de mujeres a medida que aumenta el umbral de productividad o número de trabajos publicados. Así, las mujeres representan el 40,16\% de los autores que han firmado dos trabajos, porcentaje que se reduce progresivamente hasta ser únicamente un 11,17\% las mujeres que han publicado más de nueve trabajos. Archivos de Bronconeumología es la publicación que reúne un mayor 


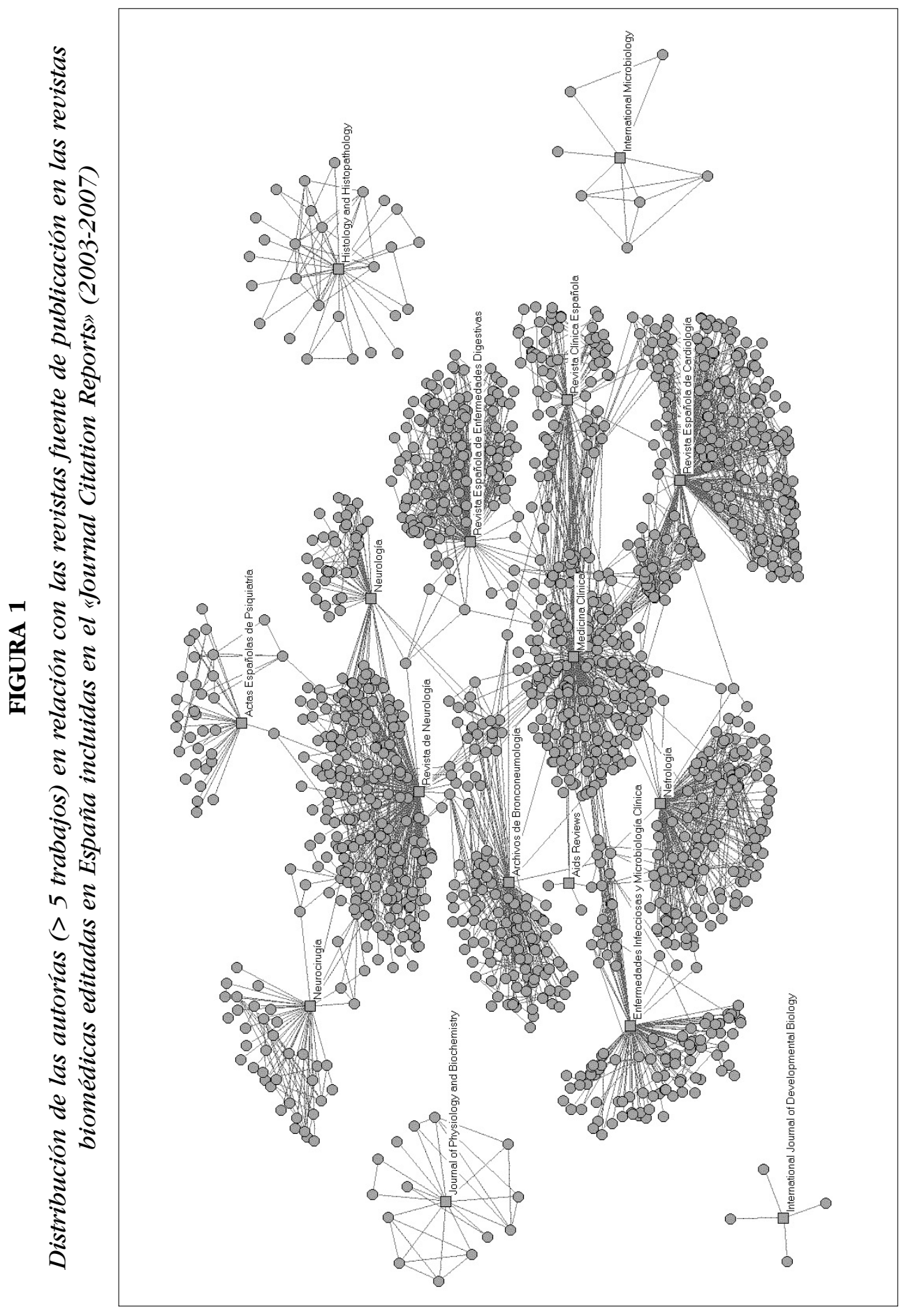


número de grandes productoras, si bien, el porcentaje es únicamente del 16,98\%, seguida por Nefrología (14,7\%), Neurología (13,95\%), Enfermedades Infecciosas y Microbiología Clínica (12,19\%), y Revista Española de Enfermedades Digestivas (11,32\%). Cabe destacar que Enfermedades Infecciosas y Microbiología Clinica es la publicación editada en español que reúne un mayor porcentaje de mujeres que han publicado dos $(54,57 \%)$ y tres trabajos $(43,18 \%)$, revista que junto a Neurología, Nefrología, Revista de Neurología y Revista Española de Enfermedades Digestivas, reúnen los porcentajes más elevados de productoras moderadas (3-9 trabajos). Por otra parte, en las revistas editadas en inglés se observa el mismo fenómeno de reducción progresiva del número de mujeres en relación con el umbral de productividad (tabla V).

De los 11.864 trabajos analizados que cuentan con afiliación institucional, un $57,97 \%(n=6.878)$ han sido realizados en colaboración y un 42,03\% ( $n=4.986)$ sin colaboración. En relación con los 6.151 artículos originales con afiliación institucional, un 66,57\% $(n=4.095)$ presentan algún tipo de colaboración institucional frente a un 33,42\% $(n=2.056)$ realizados sin colaboración. Entre las revistas editadas en español, Medicina Clínica es la que reúne un mayor porcentaje de artículos originales firmados en colaboración institucional (80,73\%), seguida por Enfermedades Infecciosas y Microbiología Clínica (75,07\%), Archivos de Bronconeumología (74,06\%) y Actas Españolas de Psiquiatría (70,09\%). Otras cuatro publicaciones se sitúan con porcentajes de colaboración por encima del 60\%: Neurología (65,45\%), Revista Clinica Española (64,05\%), Revista Española de Cardiología (62,8\%) y Revista de Neurología (61,83\%). Finalmente, Nefrología (59,56\%), Neurocirugía (52,76\%) y Revista Española de Enfermedades Digestivas $(51,83 \%)$ son las publicaciones con menores porcentajes de colaboración. En el caso de las revistas editadas en inglés, Histology and Histopathology se destaca como la publicación con un mayor grado de colaboración institucional (78,19\%), por encima de Journal of Psysiology and Biochemistry (63,33\%), International Microbiology (61,31\%), International Journal of Developmental Biology (60,26\%) y Aids Reviews (55,55\%).

En la figura 2 se recogen los porcentajes de documentos en colaboración en relación con el tipo de colaboración considerando todas las tipologías documentales. En nueve de las once revistas editadas en español predominan las colaboraciones entre diferentes instituciones del mismo país, siendo Neurocirugía y Revista Española de Enfermedades Digestivas los dos únicos casos donde la colaboración intrainstitucional se sitúa por delante de la colaboración interinstitucional, si bien, en todas las publicaciones en español la colaboración intrainstitucional tiene un peso muy destacado con porcentajes que oscilan entre el 18,79\% y el 55,78\% del total de colaboraciones. En todos los casos, la colaboración internacional es reducida, siendo Actas Españolas de Psiquiatría (11,4\%), Neurología (7,16\%), Revista Española de Cardiología (6,81\%), Revista de Neurología (6,09\%) y Archivos de Bronconeumología (5,45\%) las publicaciones con mayores porcentajes de colaboración internacional, sea ésta generada en trabajos con participación española y extranjera o con participación de dos o más países extran- 


\section{TABLA V}

Distribución porcentual desagregada por sexos y por niveles de productividad de la participación de hombres y mujeres en los trabajos publicados en las revistas biomédicas editadas en España incluidas en el «Journal Citation Reports» (2003-2007)

\begin{tabular}{|c|c|c|c|c|c|c|c|c|c|}
\hline \multirow{3}{*}{ Área } & \multirow{3}{*}{ Revistas } & \multicolumn{8}{|c|}{ Número de autores con $n$ firmas } \\
\hline & & \multicolumn{2}{|c|}{2} & \multicolumn{2}{|c|}{3} & \multicolumn{2}{|c|}{4} & \multicolumn{2}{|c|}{5} \\
\hline & & $\% \mathbf{H}$ & $\% \mathbf{M}$ & $\% \mathbf{H}$ & $\% \mathbf{M}$ & $\% \mathbf{H}$ & $\% \mathbf{M}$ & $\% \mathbf{H}$ & $\% \mathbf{M}$ \\
\hline \multirow{12}{*}{ 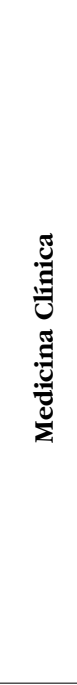 } & 1. Actas Españolas de Psiquitría & 54,17 & 45,83 & 61,82 & 38,18 & 70,97 & 29,03 & 67,86 & 32,14 \\
\hline & 2. Archivos de Bronconeumología & 62,07 & 37,93 & 64,97 & 35,03 & 69,03 & 30,97 & 69,7 & 30,3 \\
\hline & $\begin{array}{l}\text { 3. Enfermedades Infecciosas y Mi- } \\
\text { crobiología Clinica }\end{array}$ & 45,43 & 54,57 & 56,82 & 43,18 & 63,83 & 36,17 & 65,26 & 34,74 \\
\hline & 4. Medicina Clinica & 57,61 & 42,39 & 64,78 & 35,22 & 70,78 & 29,22 & 70,8 & 29,2 \\
\hline & 5. Nefrología & 64,28 & 35,71 & 62,07 & 37,93 & 60,2 & 39,79 & 57,32 & 42,68 \\
\hline & 6. Neurocirugía & 66,67 & 33,33 & 79,69 & 20,31 & 74,36 & 25,64 & 74,19 & 25,81 \\
\hline & 7. Neurología & 51,28 & 48,72 & 61,15 & 38,85 & 56,7 & 43,3 & 60,87 & 39,13 \\
\hline & 8. Revista Clínica Española & 55,95 & 44,05 & 63,31 & 36,69 & 70,06 & 29,94 & 67,86 & 32,14 \\
\hline & 9. Revista Española de Cardiología & 67,73 & 32,27 & 72,56 & 27,44 & 75,9 & 24,1 & 75,96 & 24,04 \\
\hline & $\begin{array}{l}\text { 10. Revista Española de Enferme- } \\
\text { dades Digestivas }\end{array}$ & 70,83 & 29,17 & 71,11 & 28,89 & 75,7 & 24,3 & 62,34 & 37,66 \\
\hline & 11. Revista de Neurología & 65,88 & 34,12 & 59,21 & 40,79 & 63,03 & 36,97 & 66,92 & 33,08 \\
\hline & Total $^{1}$ & 59,23 & 40,76 & 64,58 & 35,42 & 68,36 & 31,64 & 67,64 & 32,36 \\
\hline \multirow{6}{*}{ 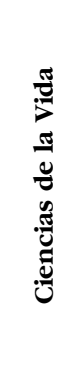 } & 12. Aids Reviews & 48 & 52 & 40 & 60 & 75 & 25 & 80 & 20 \\
\hline & 13. Histology and Histopathology & 69,23 & 30,77 & 63,02 & 36,97 & 65,96 & 34,04 & 88,23 & 11,76 \\
\hline & $\begin{array}{l}\text { 14. International Journal of Deve- } \\
\text { lopmental Biology }\end{array}$ & 73,5 & 26,49 & 53,57 & 46,43 & 64,28 & 35,71 & 66,67 & 33,33 \\
\hline & 15. International Microbiology & 56,76 & 43,24 & 75 & 25 & 50 & 50 & 63,64 & 36,36 \\
\hline & $\begin{array}{l}\text { 16. Journal of Physiology and Bio- } \\
\text { chemistry }\end{array}$ & 33,33 & 66,67 & 43,48 & 56,52 & 50 & 50 & 77,78 & 22,22 \\
\hline & Total $^{1}$ & 64,19 & 35,81 & 59,16 & 40,84 & 62,35 & 37,65 & 77,27 & 22,73 \\
\hline
\end{tabular}

H: hombres; M: mujeres. ${ }^{(1)}$ El número total de autores no se corresponde con la suma de los autores por revistas por el hecho de que los mismos autores intervienen en diferentes revistas. 


\section{TABLA V (continuación)}

\begin{tabular}{|c|c|c|c|c|c|c|c|c|c|c|c|}
\hline \multicolumn{10}{|c|}{ Número de autores con $n$ firmas } & \multirow{2}{*}{\multicolumn{2}{|c|}{$\begin{array}{c}\text { Total } \\
(>1 \text { trabajo })\end{array}$}} \\
\hline \multicolumn{2}{|c|}{6} & \multicolumn{2}{|c|}{7} & \multicolumn{2}{|c|}{8} & \multicolumn{2}{|c|}{9} & \multicolumn{2}{|c|}{$>9$} & & \\
\hline$\% \mathbf{H}$ & $\% \mathbf{M}$ & $\% \mathbf{H}$ & $\% \mathbf{M}$ & $\% \mathbf{H}$ & $\% \mathbf{M}$ & $\% \mathbf{H}$ & $\% \mathbf{M}$ & $\% \mathbf{H}$ & $\% \mathbf{M}$ & $\% \mathbf{H}$ & $\% \mathbf{M}$ \\
\hline 79,17 & 20,83 & 83,33 & 16,67 & 90 & 10 & 83,33 & 16,67 & 93,33 & 6,67 & 70,35 & 29,65 \\
\hline 68,08 & 31,91 & 77,5 & 22,5 & 90,91 & 9,09 & 78,95 & 21,05 & 83,02 & 16,98 & 68,61 & 31,39 \\
\hline 76,81 & 23,19 & 74,42 & 25,58 & 77,14 & 22,86 & 76,92 & 23,08 & 87,8 & 12,19 & 59,45 & 40,55 \\
\hline 75 & 25 & 75 & 25 & 79,65 & 20,35 & 78,95 & 21,05 & 89,84 & 10,16 & 67,32 & 32,68 \\
\hline 66,04 & 33,96 & 73,53 & 26,47 & 81,82 & 18,18 & 64,7 & 35,29 & 85,29 & 14,7 & 66,16 & 33,83 \\
\hline 83,33 & 16,67 & 80 & 20 & 87,5 & 12,5 & 75 & 25 & 95,65 & 4,35 & 79,53 & 20,46 \\
\hline 68,96 & 31,03 & 76,19 & 23,81 & 83,87 & 16,13 & 72,73 & 27,27 & 86,05 & 13,95 & 66,89 & 33,1 \\
\hline 78,09 & 21,9 & 81,82 & 18,18 & 84,61 & 15,38 & 81,58 & 18,42 & 91,33 & 8,67 & 70,94 & 29,06 \\
\hline 81,94 & 18,05 & 86,44 & 13,56 & 81,58 & 18,42 & 77,42 & 22,58 & 92,8 & 7,2 & 74,44 & 25,56 \\
\hline 76,27 & 23,73 & 78,26 & 21,74 & 74,07 & 25,92 & 66,67 & 33,33 & 88,68 & 11,32 & 73,24 & 26,76 \\
\hline 76,54 & 23,46 & 68,11 & 31,88 & 78 & 22 & 72,97 & 27,03 & 89,34 & 10,65 & 67,57 & 32,43 \\
\hline 76,42 & 23,58 & 76,42 & 23,57 & 80,43 & 19,56 & 77,78 & 22,22 & 88,54 & 11,45 & 67,07 & 32,93 \\
\hline 75 & 25 & 60 & 40 & 100 & 0 & 100 & 0 & 100 & 0 & 62,69 & 37,31 \\
\hline 75 & 25 & 50 & 50 & 75 & 25 & 100 & 0 & 91,67 & 8,33 & 68,24 & 31,76 \\
\hline 50 & 50 & 0 & 100 & 100 & 0 & 0 & 0 & 100 & 0 & 68,86 & 31,14 \\
\hline 100 & 0 & 0 & 100 & 100 & 0 & 100 & 0 & 100 & 0 & 61,61 & 38,39 \\
\hline 85,71 & 14,28 & 50 & 50 & 66,67 & 33,33 & 0 & 0 & 100 & 0 & 57,75 & 42,25 \\
\hline 76,92 & 23,08 & 50 & 50 & $\mathbf{8 1 , 2 5}$ & 18,75 & 100 & 0 & 95,65 & 4,35 & 65,51 & 34,49 \\
\hline
\end{tabular}




\section{FIGURA 2}

Distribución porcentual de los tipos de colaboración institucional en los trabajos publicados por las revistas biomédicas editadas en España incluidas en el «Journal Citation Reports»(2003-2007)

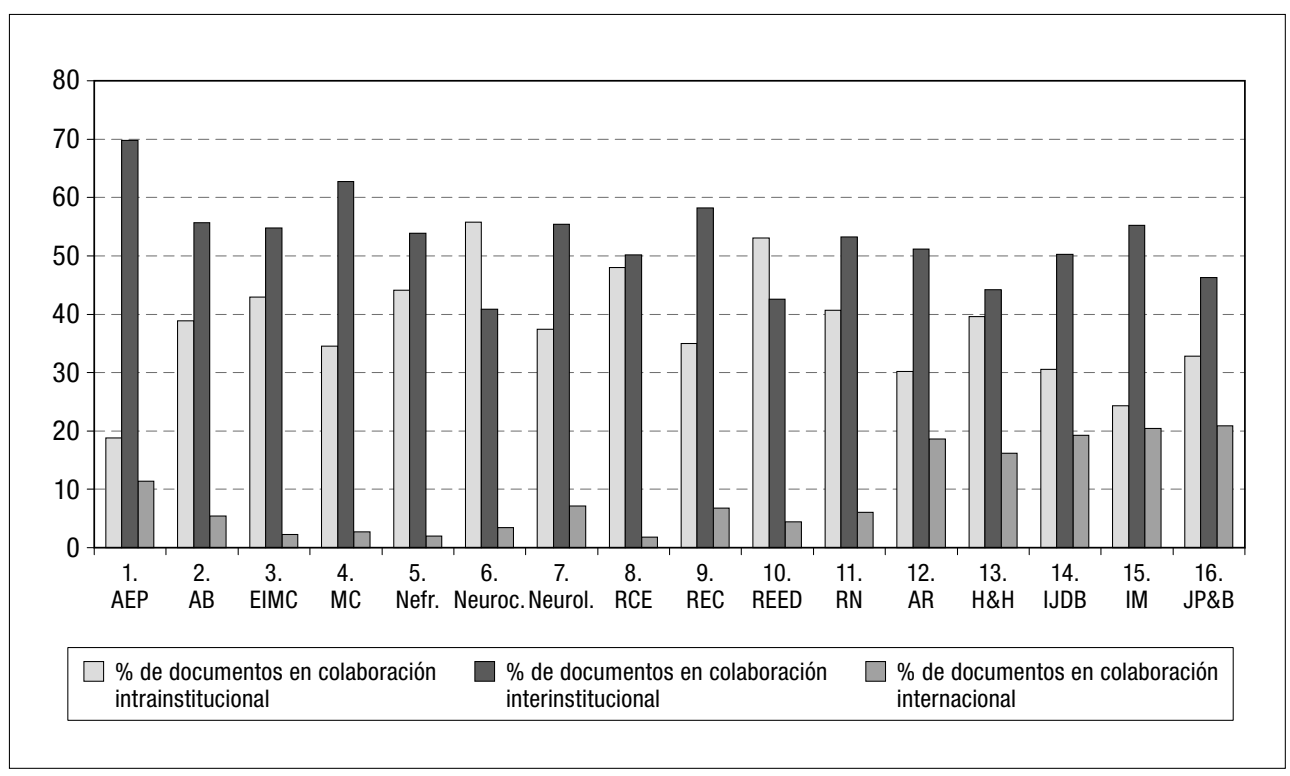

AEP (Actas Españolas de Psiquiatría), AB (Archivos de Bronconeumología), EIMC (Enfermedades Infecciosas y Microbiología Clinica), MC (Medicina Clinica), Nefr. (Nefrología), Neuroc. (Neurocirugía), Neurol. (Neurología), REC (Revista Clinica Española), REC (Revista Española de Cardiología), REED (Revista Española de Enfermedades Digestivas), RN (Revista de Neurologia), AR (Aids Reviews), H\&H (Histology and Histopathology), IJDB (International Journal of Developmental Biology), IM (International Microbiology), JP\&B (Journal of Physiology and Biochemistry).

jeros diferentes. En relación con las publicaciones editadas en inglés, también se sitúan en primer lugar las colaboraciones interinstitucionales entre diferentes centros de un mismo país, con un peso destacado de las colaboraciones intrainstitucionales, si bien en este caso, tienen un peso mucho mayor los documentos realizados en colaboración internacional, con porcentajes que oscilan entre el $16,22 \%$ y el $20,89 \%$ de las colaboraciones. En relación con las colaboraciones interinstitucionales de los trabajos en los que han participado instituciones españolas, cabe resaltar que predominan las colaboraciones con centros de la misma comunidad autónoma frente a las colaboraciones interautonómicas, con porcentajes de colaboraciones entre centros de una misma comunidad autónoma que oscilan entre el 69,06\% de Actas Españolas de Psiquiatría y el 92,68\% de colaboraciones de Neurocirugía en el caso de las revistas editadas en español; y el 60\% de Aids Reviews y el 81,25\% de International Journal of Developmental Biology en el caso de las revistas editadas en inglés. 
Considerando todas las tipologías documentales, 5.980 trabajos no han recibido ninguna cita (48,48\%), de los que 2.383 son artículos originales $(36,93 \%)$, presentando las revistas editadas en español porcentajes más altos de trabajos no citados según se recoge en la figura 3. El conjunto de artículos analizados han recibido 25.102 citas, de las que 15.586 corresponden a artículos originales. Entre las revistas editadas en español, Medicina Clínica es la que reúne el mayor número de citas en términos absolutos $(n=3.342)$, seguida por Revista Española de Cardiología ( $n=2.690)$, Revista de Neurologia $(n=2.343)$, Archivos de Bronconeumología ( $n=1.585$ ) y Enfermedades Infecciosas y Microbiología Clinica $(n=1.272)$, situándose el resto de publicaciones por debajo de las 1.000 citas. Histology and Histopathology encabeza la citación en términos absolutos de las publicaciones editadas en inglés $(n=4.288)$, seguida por International Journal of Developmental Biology $(n=4.287)$ e International Microbiology $(n=1.146)$ (tabla VI).

\section{FIGURA 3}

Porcentaje de artículos originales no citados (2003-2005) en los trabajos publicados en las revistas biomédicas editadas en España incluidas en el «Journal Citation Reports»

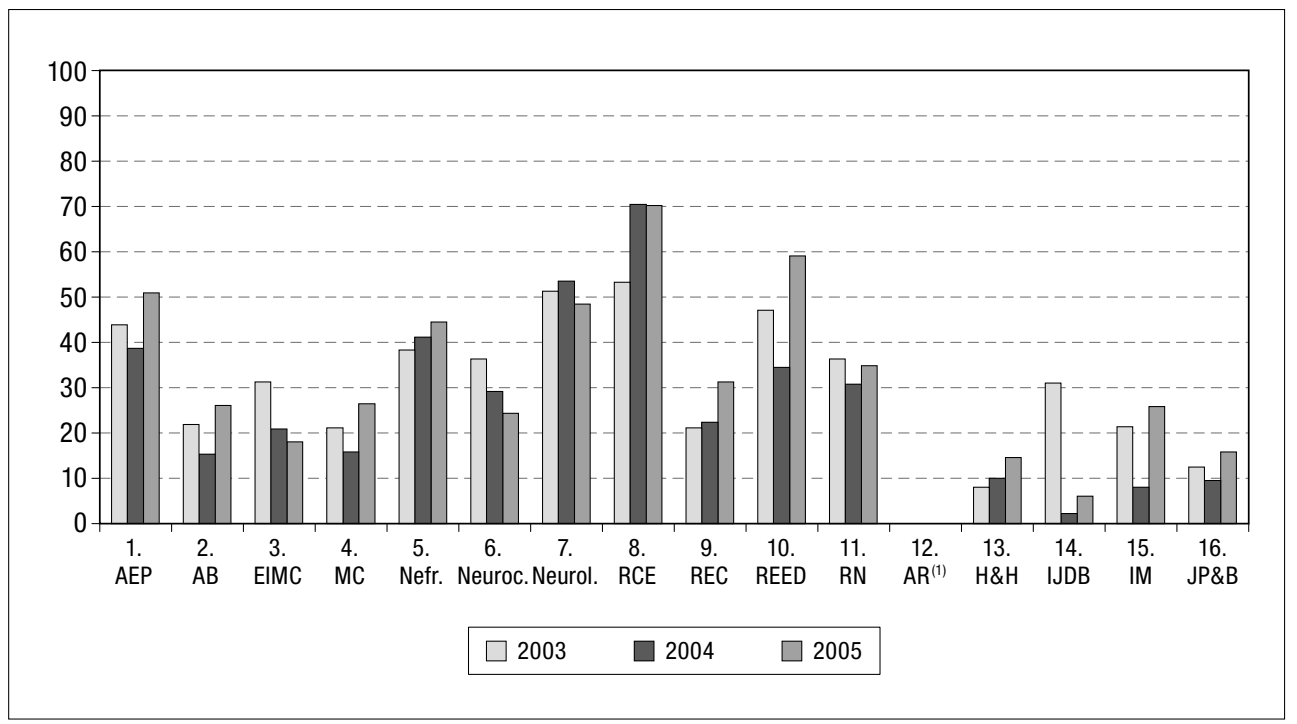

(1) Aids Reviews (AR) presenta un carácter irregular, ha sido indizada en SCI-Expanded a partir de 2004, y ha publicado únicamente los años 2004 y 2005 revisiones. Ver nombres de revistas en figura 2.

Los artículos originales de cuatro publicaciones editadas en español presentan una media de citas por trabajo por encima de dos los años 2003, 2004 y 2005 según se muestra en la figura 4: Revista Española de Cardiología (4,19 en 


\section{TABLA VI}

Número de citas recibidas por los trabajos publicados en las revistas biomédicas estudiadas

\begin{tabular}{|c|c|c|c|c|c|c|c|c|}
\hline \multirow[b]{2}{*}{ Área } & \multirow[b]{2}{*}{ Revista } & \multicolumn{7}{|c|}{ Todos los documentos } \\
\hline & & 2003 & 2004 & 2005 & 2006 & 2007 & Total & $\begin{array}{l}\text { Media } \\
\text { de citas/ } \\
\text { trab. } \pm \text { DS }\end{array}$ \\
\hline \multirow{12}{*}{ 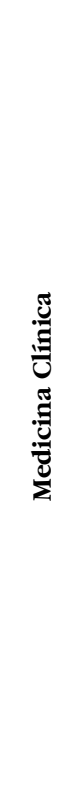 } & $\begin{array}{l}\text { 1. Actas Españolas de Psi- } \\
\text { quiatría }\end{array}$ & 83 & 79 & 70 & 32 & 23 & 287 & $0,95 \pm 1,79$ \\
\hline & $\begin{array}{l}\text { 2. Archivos de Bronconeu- } \\
\text { mología }\end{array}$ & 345 & 422 & 360 & 284 & 174 & 1.585 & $2,23 \pm 2,87$ \\
\hline & $\begin{array}{l}\text { 3. Enfermedades Infecciosas } \\
\text { y Microbiología Clinica }\end{array}$ & 311 & 344 & 328 & 184 & 105 & 1.272 & $1,58 \pm 3,19$ \\
\hline & 4. Medicina Clínica & 977 & 836 & 841 & 450 & 238 & 3.342 & $1,25 \pm 3,01$ \\
\hline & 5. Nefrología & 149 & 148 & 155 & 114 & 54 & 620 & $1,02 \pm 2,28$ \\
\hline & 6. Neurocirugía & 71 & 87 & 76 & 27 & 29 & 290 & $0,93 \pm 1,41$ \\
\hline & 7. Neurología & 151 & 113 & 138 & 125 & 73 & 600 & $1,13 \pm 1,94$ \\
\hline & 8. Revista Clínica Española & 118 & 74 & 68 & 78 & 47 & 385 & $0,39 \pm 0,92$ \\
\hline & $\begin{array}{l}\text { 9. Revista Española de Car- } \\
\text { diología }\end{array}$ & 693 & 677 & 572 & 439 & 309 & 2.690 & $2,51 \pm 4,8$ \\
\hline & $\begin{array}{l}\text { 10. Revista Española de En- } \\
\text { fermedades Digestivas }\end{array}$ & 85 & 198 & 130 & 157 & 66 & 636 & $0,8 \pm 1,66$ \\
\hline & 11. Revista de Neurología & 703 & 693 & 454 & 272 & 221 & 2.343 & $1,27 \pm 1,73$ \\
\hline & Total & 3.686 & 3.671 & 3.192 & 2.162 & 1.339 & 14.050 & $1,32 \pm 2,77$ \\
\hline \multirow{6}{*}{ 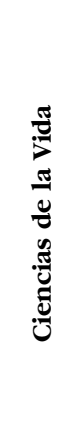 } & 12. Aids Reviews ${ }^{(1)}$ & 0 & 364 & 230 & 154 & 58 & 806 & $4,85 \pm 7,49$ \\
\hline & $\begin{array}{l}\text { 13. Histology and Histopa- } \\
\text { thology }\end{array}$ & 1.154 & 1.221 & 856 & 686 & 371 & 4.288 & $6,23 \pm 7,45$ \\
\hline & $\begin{array}{l}\text { 14. International Journal of } \\
\text { Developmental Biology }\end{array}$ & 748 & 2.046 & 928 & 405 & 160 & 4.287 & $8,86 \pm 13,09$ \\
\hline & $\begin{array}{l}\text { 15. International Microbiolo- } \\
\text { gy }\end{array}$ & 278 & 396 & 262 & 165 & 45 & 1.146 & $5,76 \pm 8,65$ \\
\hline & $\begin{array}{l}\text { 16. Journal of Physiology and } \\
\text { Biochemistry }\end{array}$ & 200 & 139 & 100 & 70 & 16 & 525 & $3,75 \pm 5,92$ \\
\hline & Total & 2.380 & 4.166 & 2.376 & 1.480 & 650 & 11.052 & $6,59 \pm 9,59$ \\
\hline
\end{tabular}

DS: Desviación estándar. ${ }^{(1)}$ Aids Reviews presenta un carácter irregular, ha sido indizada en SCI-Expanded a partir de 2004, y ha publicado únicamente los años 2004 y 2005 revisiones. 


\section{TABLA VI (continuación)}

\begin{tabular}{|c|c|c|c|c|c|c|c|c|}
\hline \multirow[b]{2}{*}{ Área } & \multirow[b]{2}{*}{ Revista } & \multicolumn{7}{|c|}{ Artículos originales } \\
\hline & & 2003 & 2004 & 2005 & 2006 & 2007 & Total & $\begin{array}{l}\text { Media } \\
\text { de citas/ } \\
\text { trab. } \pm \text { DS }\end{array}$ \\
\hline \multirow{12}{*}{ 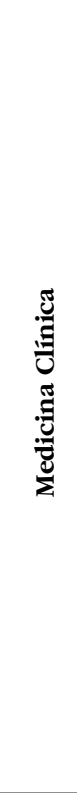 } & $\begin{array}{l}\text { 1. Actas Españolas de Psi- } \\
\text { quiatría }\end{array}$ & 69 & 72 & 69 & 29 & 19 & 258 & $1,13 \pm 1,97$ \\
\hline & $\begin{array}{l}\text { 2. Archivos de Bronconeu- } \\
\text { mología }\end{array}$ & 270 & 339 & 243 & 226 & 144 & 1.222 & $2,8 \pm 3,08$ \\
\hline & $\begin{array}{l}\text { 3. Enfermedades Infecciosas } \\
\text { y Microbiología Clinica }\end{array}$ & 229 & 215 & 241 & 145 & 53 & 883 & $2,51 \pm 3,79$ \\
\hline & 4. Medicina Clinica & 640 & 586 & 576 & 252 & 131 & 2.185 & $2,43 \pm 4,49$ \\
\hline & 5. Nefrología & 122 & 123 & 132 & 86 & 48 & 511 & $1,35 \pm 2,62$ \\
\hline & 6. Neurocirugía & 57 & 75 & 61 & 18 & 19 & 230 & $1,09 \pm 1,45$ \\
\hline & 7. Neurología & 127 & 85 & 91 & 81 & 45 & 429 & $1,15 \pm 1,93$ \\
\hline & 8. Revista Clínica Española & 89 & 56 & 53 & 61 & 38 & 297 & $0,67 \pm 1,2$ \\
\hline & $\begin{array}{l}\text { 9. Revista Española de Car- } \\
\text { diología }\end{array}$ & 495 & 420 & 313 & 243 & 204 & 1.675 & $3,28 \pm 5,84$ \\
\hline & $\begin{array}{l}\text { 10. Revista Española de En- } \\
\text { fermedades Digestivas }\end{array}$ & 73 & 175 & 95 & 113 & 49 & 505 & $1,29 \pm 2,03$ \\
\hline & 11. Revista de Neurología & 558 & 455 & 306 & 140 & 133 & 1.592 & $1,42 \pm 1,8$ \\
\hline & Total & 2.729 & 2.601 & 2.180 & 1.394 & 883 & 9.787 & $1,83 \pm 3,33$ \\
\hline \multirow{6}{*}{ 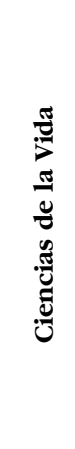 } & 12. Aids Reviews ${ }^{(1)}$ & 0 & - & - & 87 & - & 87 & $2,41 \pm 4,09$ \\
\hline & $\begin{array}{l}\text { 13. Histology and Histopa- } \\
\text { thology }\end{array}$ & 463 & 432 & 307 & 232 & 191 & 1.625 & $3,76 \pm 4,01$ \\
\hline & $\begin{array}{l}\text { 14. International Journal of } \\
\text { Developmental Biology }\end{array}$ & 503 & 1.399 & 672 & 322 & 114 & 3.010 & $7,84 \pm 11,16$ \\
\hline & $\begin{array}{l}\text { 15. International Microbiolo- } \\
\text { gy }\end{array}$ & 138 & 221 & 221 & 105 & 41 & 726 & $5,11 \pm 7,79$ \\
\hline & $\begin{array}{l}\text { 16. Journal of Physiology and } \\
\text { Biochemistry }\end{array}$ & 139 & 71 & 80 & 53 & 8 & 351 & $2,88 \pm 3,92$ \\
\hline & Total & 1.243 & 2.123 & 1.280 & 799 & 354 & 5.799 & $5,2 \pm 7,93$ \\
\hline
\end{tabular}




\section{FIGURA 4}

Media de citas por trabajo de los artículos originales (2003-2005) en los trabajos publicados en las revistas biomédicas editadas en España incluidas en el «Journal Citation Reports»

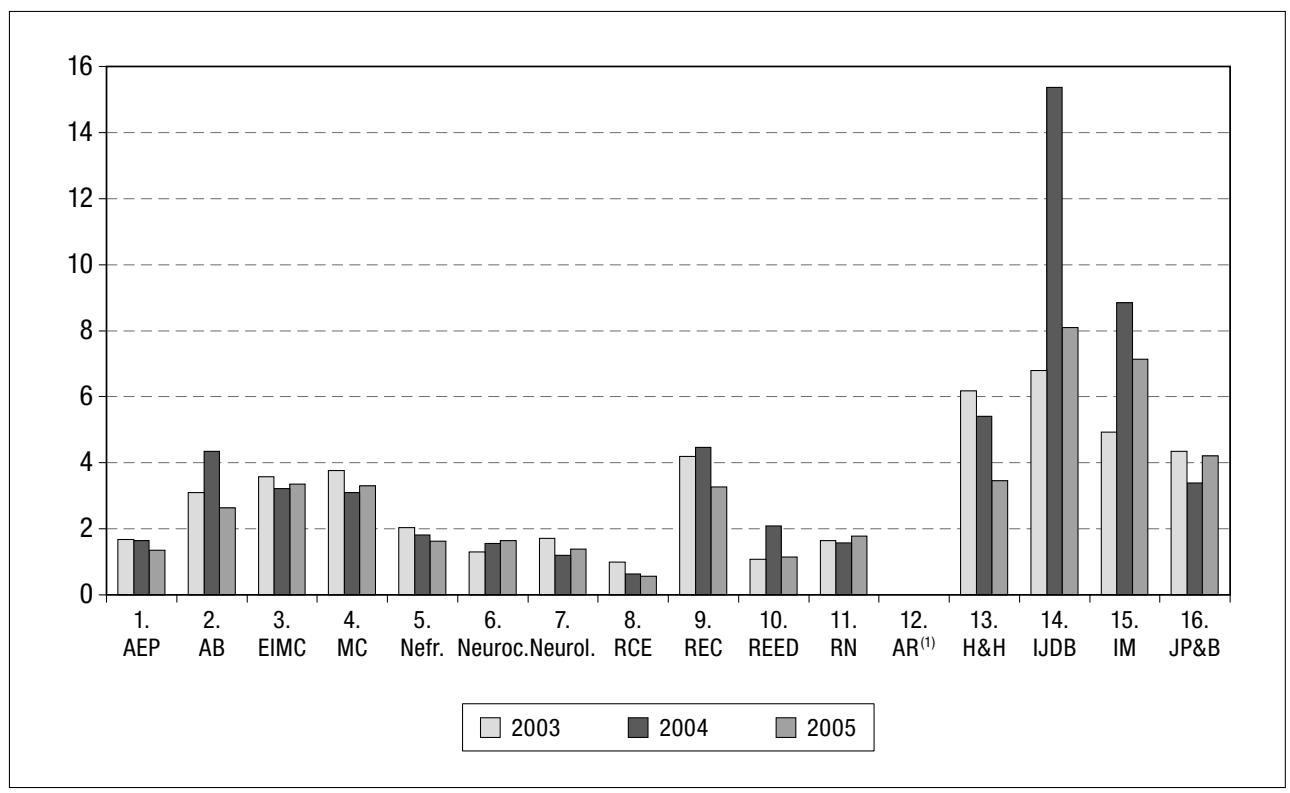

(1) Aids Reviews (AR) presenta un carácter irregular, ha sido indizada en SCI-Expanded a partir de 2004, y ha publicado únicamente los años 2004 y 2005 revisiones. Ver nombres de revistas en figura 2.

2003, 4,47 en 2004 y 3,26 en 2005), Archivos de Bronconeumología (3,1 en 2003, 4,35 en 2004 y 2,64 en 2005), Enfermedades Infecciosas y Microbiología Clinica (3,57 en 2003, 3,21 en 2004 y 3,35 en 2005) y Medicina Clínica (3,76 en 2003, 3,1 en 2004 y 3,31 en 2005). Las revistas editadas en inglés presentan medias de citas/trabajo mucho más elevadas, siempre por encima de tres.

En la figura 5 se presenta la evolución de los factores de impacto en el JCR de las revistas editadas en español (2003-2007) y en la figura 6 de las revistas editadas en inglés (2003-2007). La evolución diacrónica de los factores de impacto permite apreciar un importante crecimiento en los factores de impacto de Revista Española de Cardiología y de Archivos de Bronconeumología a lo largo del período analizado, situándose por encima del resto de publicaciones, especialmente la primera de ellas. La tendencia general que presentan todas ellas es, no obstante, de un incremento lento pero sostenido del factor de impacto. En cambio, en relación con las revistas editadas en inglés, cabe destacar que la tendencia de crecimiento se rompe en el año 2007, ya que cuatro de las cinco revistas han visto reducido su factor este último año. Finalmente, cabe resaltar que cinco de las revistas editadas en español han mejorado su posición en el 


\section{FIGURA 5}

Evolución de los factores de impacto de las revistas biomédicas editadas en España en español incluidas en el «Journal Citation Reports».

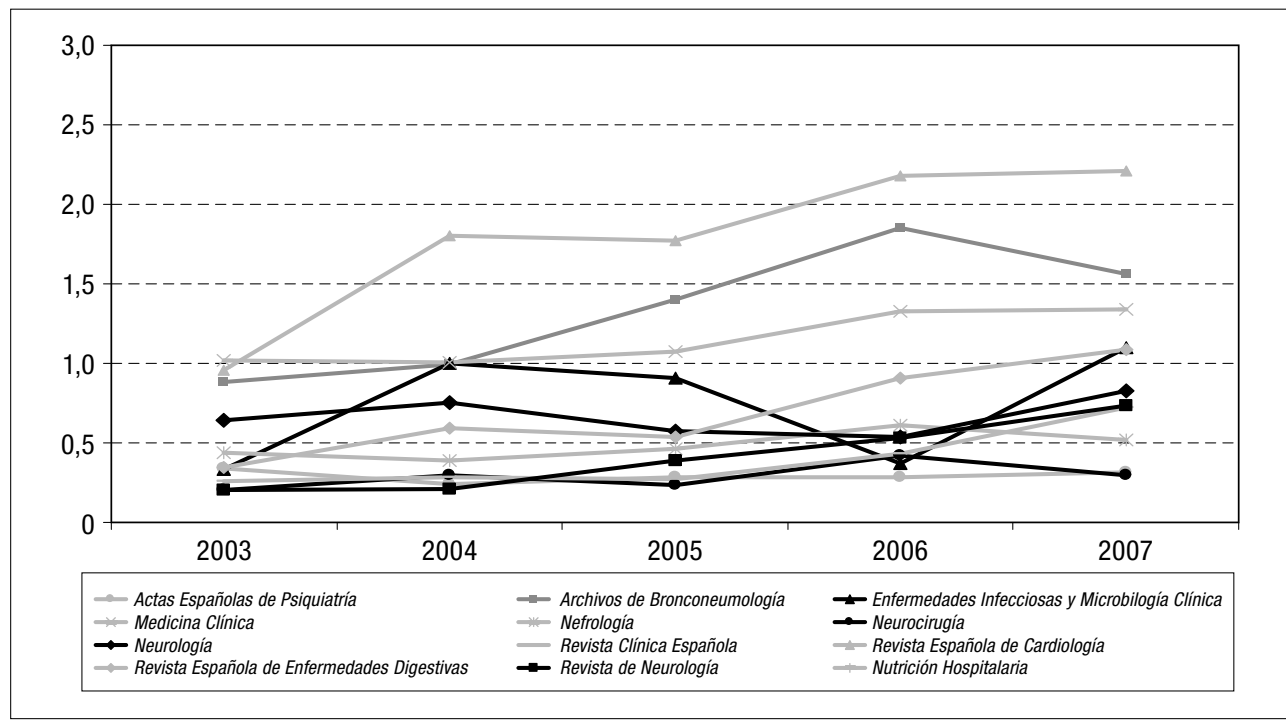

Gris claro: revistas con ediciones en inglés; negro: revistas editadas únicamente en español.

\section{FIGURA 6}

Evolución de los factores de impacto de las revistas biomédicas editadas en España en inglés incluidas en el "Journal Citation Reports».

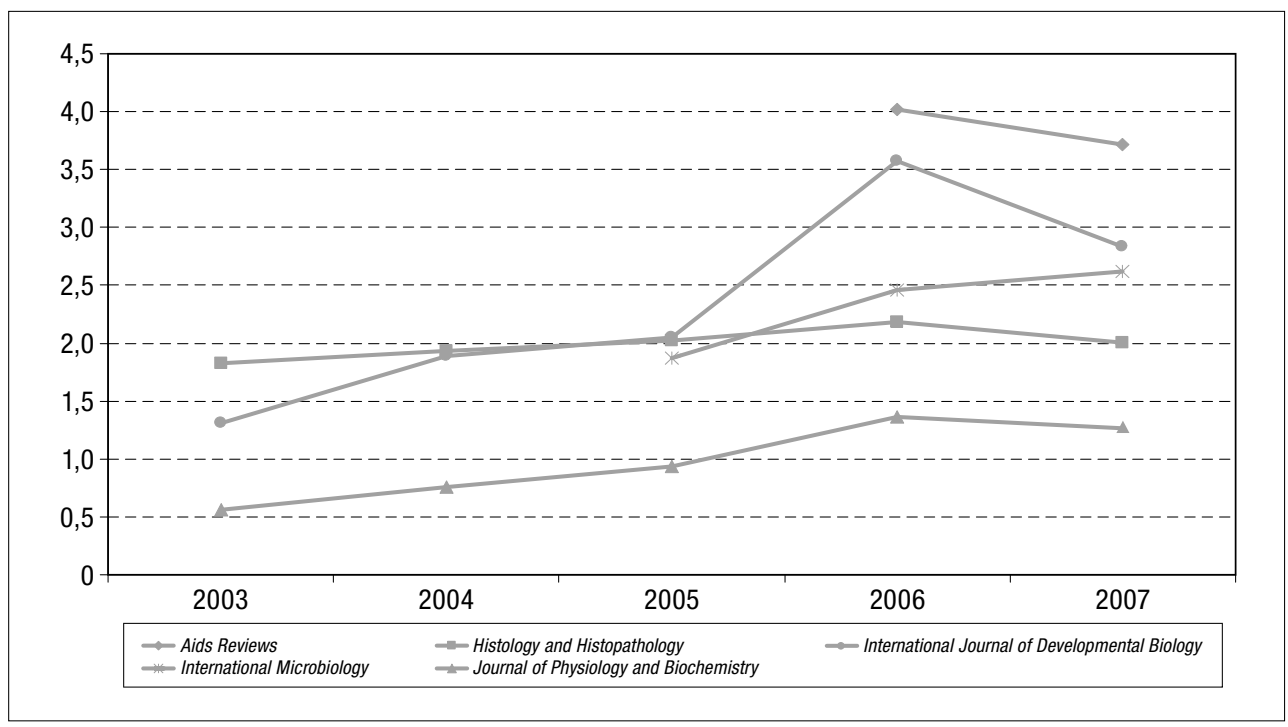


ranking de revistas del JCR del área a la que se adscriben entre el primer y el último año del período analizado: Revista Clínica Española (ha pasado del puesto 93 de 102 revistas al puesto 72 de 100 revistas), Revista Española de Cardiología (puesto 48 de 70 revistas a puesto 28 de 74 revistas), Revista de Neurología (puesto 132 de 135 a puesto 126 de 146), Neurocirugía (puesto 134 de 141 a puesto 131 de 139) y Archivos de Bronconeumología (puesto 27 de 31 a puesto 26 de 34). Revista Española de Enfermedades Digestivas mantiene su posición (puesto 45 de 47 a puesto 45 de 50), mientras que el resto de revistas han perdido diferentes puestos, pese al incremento en términos absolutos del factor de impacto de algunas de ellas.

\section{Discusión}

Uno de los aspectos más significativos del estudio realizado es que las revistas editadas en español del área de la Medicina Clínica canalizan una parte importante de la producción científica española en sus respectivas disciplinas según la clasificación de revistas del JCR, destacando sobre todo las áreas con presencia de más de una revista, ya que las dos revistas de Medicina General e Interna totalizan el 81,22\% de los trabajos españoles del área, y las dos revistas de Neurología Clínica el 43,23\% de los trabajos firmados por, al menos, una institución española en esta disciplina. Esta situación contrasta, sin embargo, con el hecho de que en el período de realización del estudio solamente nueve de las 44 disciplinas de la clasificación del JCR encuadrables en el área de la Medicina Clínica contaban con la presencia de alguna revista española, con lo que los investigadores de otras áreas debían recurrir necesariamente a publicar sus trabajos en revistas extranjeras para alcanzar un adecuado rendimiento de su trabajo, al ser las bases de datos de Thomson Reuters (antes Thomson-ISI) el principal referente a nivel curricular y evaluativo (González Alcaide y otros, 2008; Medina Casaubón y otros, 2008). Esta circunstancia se ha visto paliada por la reciente inclusión de revistas españolas en la base de datos SCI-Expanded en otras diez disciplinas, así como por la inclusión de otras revistas en algunas áreas que ya contaban con alguna publicación española, con lo que transcurridos dos años pasarán a tener factor de impacto, lo que supone un importante impulso para la difusión internacional de los trabajos publicados por las revistas españolas de estas áreas (Aleixandre Benavent, 2009). No obstante, es importante advertir que sigue sin estar resuelta de forma absoluta la escasa representatividad de revistas fuente españolas en algunas destacadas disciplinas y especialidades, en las que la contribución científica española a la producción científica mundial es importante, como la Anestesiología; Dermatología; Geriatría y Gerontología; Hematología; Oftalmología; Ortopedia; Otorrinolaringología; Diagnóstico por la Imagen, Medicina Nuclear y Radiología; o Reumatología, entre otras (González Alcaide y otros, 2009b). En relación con las Ciencias de la Vida, solamente ocho de las 19 disciplinas cuentan con presencia española, con un predominio de las revistas edita- 
das en inglés (siete revistas), no habiéndose producido ninguna incorporación reciente de revistas, a diferencia de lo que ha ocurrido con la Medicina Clínica y con otras áreas de conocimiento de las Ciencias Sociales y Humanas, tratándose de publicaciones que presentan aportes reducidos en relación con la producción científica española de esas disciplinas y con una partición española mucho más reducida.

La dualidad observada en relación con las revistas biomédicas editadas en España coincide también en gran medida con la orientación de las investigaciones: clínica o básica, en el caso de las revistas de Ciencias de la Vida. Muchas de las revistas editadas exclusivamente en inglés que se adscriben a disciplinas de las Ciencias de la Vida, han surgido en el ámbito universitario y en fechas más o menos recientes. Se trata de publicaciones que han adoptado estrategias editoriales de publicación orientadas a conseguir elevados índices de impacto y una publicación internacional de prestigio, lo que se pone de manifiesto por ejemplo en la publicación de un número reducido de trabajos, en la ausencia de cartas y editoriales o en la publicación mayoritaria de revisiones. En cambio, la mayor parte de las revistas en español están editadas o son el órgano oficial de sociedades médicas, tratándose de revistas clínicas cuyo origen se remonta en ocasiones varias décadas, constituyendo un importante instrumento de cohesión para la comunidad a la que representan. Así se pone de manifiesto en el hecho de que constituyen un importante foro de expresión, ya que a través de los editoriales se pueden exponer los más variados asuntos relacionados con la actividad médica, y también opiniones que pueden marcar las líneas éticas, políticas, económicas, legales o docentes a seguir; y que son un vehículo dinámico de comunicación científica, lo que queda reflejado en el importante número de cartas recogidas en la práctica totalidad de las publicaciones. Estas revistas han debido afrontar, sin embargo, el hecho de que constituyen algo más que un vehículo de comunicación científica, ya que son también instrumentos de evaluación, ya que el mayor o menor "atractivo" de las mismas para la comunidad científica, medido en términos de factor de impacto, condiciona el número y la calidad de los trabajos que reciben, y en última instancia, su propia supervivencia. En relación con esta condición, desde los comités editoriales de estas revistas se ha concedido con carácter general una gran importancia al factor de impacto, tratando de buscar el difícil equilibrio existente en ocasiones entre la necesidad de disponer de un buen factor de impacto y el hecho de orientar la política editorial de la revista a servir a la sociedad científica a la que representan y a sus lectores (Alfonso, 2009; Matías-Guiu y García Ramos, 2008).

La dualidad de la literatura difundida en las revistas biomédicas editadas en España queda reflejada además de a través de la productividad y las tipologías documentales preferentes de publicación, en otros indicadores bibliométricos, como la distribución de la participación en los trabajos por sector institucional, con una presencia mayoritaria de los centros asistenciales hospitalarios en las revistas en español, siendo por tanto estas publicaciones el canal de difusión de la actividad clínica asistencial y de la investigación desarrollada en los centros 
hospitalarios; frente a la investigación básica, más vinculada a las universidades y a los centros de investigación, cuya participación es mayoritaria en el caso de las revistas editadas en inglés, dualidad que está reflejando algunas de las principales carencias que se atribuyen al sistema científico y tecnológico español y en particular a la investigación biomédica: la escasa integración entre la investigación básica y clínica y las deficiencias relativas a la transferencia tecnológica y la aplicabilidad de los resultados derivados de las investigaciones científicas (Escudero Gómez y otros, 2008; Fundación Española para la Ciencia y la Tecnología, 2005; Rodés y Trilla, 1999; Soriguer Escofet, 2009).

En torno a cada una de las revistas científicas editadas en español analizadas, se articula una comunidad de investigadores españoles que utilizan las mismas como vehículo habitual para dar a conocer los resultados de sus investigaciones; en cambio, las revistas editadas en inglés reúnen un menor número de grupos, siendo la única excepción Histology and Histopathology, que aún siguiendo las mismas estrategias de publicación que el resto de revistas, sí que es el canal de difusión de las investigaciones para un importante número de grupos españoles. En este sentido, en relación con las áreas en las que existe más de una revista, cabe resaltar el hecho de que cada una de ellas tiene una "comunidad estable" de investigadores y que solamente un número reducido de los mismos participan de forma habitual en ambas revistas, pese a los vínculos disciplinares, geográficos e idiomáticos compartidos por ambas revistas fuente, característica en la que pueden influir las preferencias de los autores a la hora de enviar sus manuscritos (Frank, 1994), o bien responder al hecho de que las publicaciones presenten una diferente orientación o subespecialización, pese a englobarse dentro de una misma disciplina (Matías-Guiu y García Ramos, 2008). Los elevados índices de transitoriedad que presentan las revistas editadas en inglés, confirma el hecho que no existe una comunidad de investigadores estable articulada en torno a las mismas que las utilicen como canal habitual para la difusión de sus investigaciones. Asimismo, los elevados índices de transitoriedad de las revistas de la categoría Medicina General e Interna en el caso de las revistas editadas en español y el hecho de que sean las únicas publicaciones que comparten un número destacado de autores con revistas de otras especialidades, revela el carácter generalista de estas publicaciones.

El análisis de la participación de la mujer en las actividades científicas constituye un ámbito de gran relevancia social, intelectual y política (European Commissión, 2006). En este sentido, pese a la incorporación de la mujer al mundo laboral, con una creciente presencia de la misma en los ámbitos académico y científico, numerosos estudios han alertado de la existencia de diversos factores que contribuyen a que su representación sea baja en determinados estamentos, sobre todo los relacionados con cargos de alta jerarquía y responsabilidad, habiéndose llegado a referir en el ámbito de la medicina que se ha pasado de la incorporación a la discriminación de la mujer (Arrizabalaga y Valls Llobet, 2005). Diversos trabajos referidos a disciplinas y especialidades como el Abuso de Sustancias (González Alcaide y otros, 2007), las Enfermedades Infecciosas y Micro- 
biología Clínica (Aleixandre Benavent y otros, 2007b), la Neurología (Aleixandre Benavent y otros, 2007a), la Psiquiatría (Alonso Arroyo y otros, 2008) o la Cardiología (González Alcaide y otros, 2009a), han puesto de manifiesto el menor grado de productividad de las mujeres, que ahora se puede hacer extensible a otras áreas analizadas, como la Medicina General o Interna, el Aparato Respiratorio, la Urología y Nefrología o la Gastroenterología y Hepatología. No obstante, es importante señalar que se ha observado un incremento del grado de participación de las mujeres en las publicaciones científicas. Así, en Enfermedades Infecciosas y Microbiología Clínica, se ha pasado de no existir ninguna mujer con nueve trabajos y ninguna gran productora en el período 2001-2005 a representar en el período 2003-2007 un 23,08\% y un 12,19\% de los autores, respectivamente (Aleixandre Benavent y otros, 2007b); y en Revista Española de Cardiologia, el porcentaje de autoras con 9 trabajos ha pasado de ser un 16,67\% en el período 2002-2006 a un 22,58\% en el presente estudio, mientras que el porcentaje de grandes productoras ha ascendido de un 4,08\% a un 7,2\% (González Alcaide y otros, 2009a), valores que en cualquier caso, continúan estando alejados de la paridad y de los porcentajes de mujeres que cursan estudios universitarios y obtienen el grado de doctor, por lo que resulta fundamental realizar un seguimiento tratando de identificar y eliminar cualquier barrera discriminatoria y adoptar políticas que aseguren que las mujeres puedan incorporarse y desarrollar su carrera científica en condiciones de igualdad en relación con los hombres.

El principal aspecto que cabe resaltar respecto a la colaboración científica, es el importante peso que tiene, en las revistas editadas en español, la colaboración interinstitucional entre instituciones de la misma comunidad autónoma y también la colaboración intrainstitucional, con una reducida colaboración internacional, pese a las numerosas iniciativas de los organismos gestores de las políticas científicas que tratan de fomentar la creación de redes cooperativas y la colaboración internacional. En ello incide, sin duda, el hecho de que la investigación clínica presenta una marcada vinculación nacional y de carácter local, derivado del hecho de que se estudian casos concretos de pacientes en los centros hospitalarios frente al conocimiento de carácter abstracto y universal de la investigación básica (Pesquero Franco y Muñoz-Alonso López, 1997), pero también otros factores, como el escaso reconocimiento que se ha otorgado y que sigue otorgando a las publicaciones españolas, con lo que los trabajos de mayor impacto de carácter internacional se publican preferentemente en revistas extranjeras (Jiménez Contreras, 1992); o el escaso reconocimiento e incentivos que tienen los investigadores clínicos (Bruguera, 2001; Sánchez Capelo, 2003; Praga Terente, 2008). En este sentido, desde áreas como la Neurología se ha apuntado que algunos de los principales desafíos pendientes en el ámbito de la biomedicina son la necesidad de impulsar la colaboración interdisciplinaria y la creación de redes de colaboración entre neurocientíficos básicos, clínicos y epidemiológicos, así como la necesidad de fomentar las publicaciones en español y la colaboración institucional entre centros españoles y latinoamericanos, que es singularmente escasa pese a los vínculos históricos, culturales y lingüís- 
ticos (Aleixandre Benavent y otros, 2007c; González de Dios y otros, 2007a; González de Dios y otros, 2007b).

En relación con los indicadores de citación, las publicaciones en inglés reúnen una media considerablemente más elevada de citas por trabajo $(5,2$ frente a 1,8 en el caso de los artículos originales) y un menor número de trabajos no citados (el 19,98\% frente al 40,47\% de los artículos originales publicados en revistas en español), lo que cabe atribuir a factores como las estrategias editoriales en relación con el número y tipologías documentales de los trabajos publicados; a la propia utilización del inglés, que se ha convertido en la lingua franca de publicación científica en las ciencias naturales y, en general, en la investigación básica, favoreciendo su adopción el grado de citación en estos ámbitos (Olmeda Gómez y otros, 2008); y al hecho de que se trata de las revistas con un mayor grado de colaboración internacional, variable que numerosos estudios han correlacionado positivamente con el grado de citación (Granda Orive y otros, 2007; De Filippo y otros, 2008; Rousseau, 2000). No obstante, a la hora de realizar análisis comparativos, hay que tener presente la posible incidencia de factores como el diferente tamaño y las diferentes características y comportamientos de citación existentes en relación con las áreas de conocimiento (Ciencias de la Vida y Medicina Clínica) y entre unas disciplinas y otras, lo que debe servir además para alertar acerca de la complejidad de la Ciencia y de aplicar criterios generalistas en los procesos evaluativos (González Alcaide y otros, 2009b). En relación con la adopción del inglés en el área de la Medicina Clínica, algunos estudios han puesto de manifiesto que su utilización no presupone necesariamente un aumento del grado de citación (Bracho-Riquelme y otros, 1997), habiéndose defendido que la utilización del español puede resultar beneficiosa para la comunidad científica hispanohablante (Aleixandre Benavent y otros, 2007c; González de Dios y otros, 2007a; González de Dios y otros, 2007b), además de evitar el reduccionismo que comporta toda homogeneización lingüística realizada sin tener en cuenta las características de la comunidad científica de una disciplina o área de conocimiento (Gingras, 1984). El estudio realizado no permite realizar inferencias en relación con la traducción al inglés de los contenidos de las publicaciones editadas en español, aspecto que podría abordarse en futuros trabajos. Sí que parece deducirse en cambio, que la integración de los investigadores en grupos de investigación favorece el grado de citación, ya que en nueve de las once revistas editadas en español analizadas, los trabajos firmados por investigadores integrados en alguno de los grupos identificados presentan un mayor porcentaje de trabajos citados o una media más elevada de citas por trabajo que aquellos trabajos firmados por autores no integrados en ninguno de los grupos; también las publicaciones con los índices de firmas por trabajo más elevados son las que presentan los mayores factores de impacto; y en ocho de las once publicaciones editadas en español, los trabajos en los que participa alguna mujer presentan igualmente, un mayor porcentaje de trabajos citados o una media más elevada de citas por trabajo. Futuros estudios deben profundizar en el análisis de la relación existente 
entre todas estas variables y el grado de citación de las publicaciones científicas (Callahan y otros, 2002).

\section{Bibliografía}

Alfonso, F. (2009). Revistas biomédicas españolas: relevancia académica, impacto científico o factor de impacto. ¿Qué es lo que importa? Revista de Neurología, vol. 48 (3), 113-116.

Aleixandre Benavent, R.; Alonso Arroyo, A.; González Alcaide, G.; González de Dios, J.; Pérez Sempere, A., y Valderrama Zurián, J. C. (2007a). Análisis de género de los artículos publicados en Revista de Neurología durante el quinquenio 2002-2006. Revista de Neurología, vol. 45 (3), 137-143.

Aleixandre Benavent, R.; González Alcaide, G.; Alonso Arroyo, A.; Castellano Gómez, M., y Valderrama Zurián, J. C. (2007b). Valoración de la paridad en la autoría de los artículos publicados en la revista Enfermedades Infecciosas y Microbiología Clínica durante el quinquenio 2001-2005. Enfermedades Infecciosas y Microbiología Clínica, vol. 25 (10), 619-626.

Aleixandre Benavent, R.; Valderrama Zurián, J. C.; Alonso Arroyo, A.; Miguel Dasit, A.; González de Dios, J., y Granda Orive, J. I. de. (2007c). Español frente a inglés como idioma de publicación y factor de impacto de Neurología. Neurología, vol. 22 (1), 19-26.

Aleixandre Benavent, R. (2009). Factor de impacto, competencia comercial entre "Thomson Reuters" y "Elsevier", y crisis económica. En: Baiget, T. (director). Anuario Thinkepi: análisis de tendencias en información y documentación. Barcelona; El Profesional de la Información, p. 27-29.

Alonso Arroyo, A.; González Alcaide, G.; Bolaños Pizarro, M.; Castelló Cogollos, L.; Valderrama Zurián, J. C., y Aleixandre Benavent, R. (2008). Análisis de género de los trabajos publicados en la revista Actas Españolas de Psiquiatría (1999-2006). Actas Españolas de Psiquiatría, vol. 36 (6), 314-322.

Arrizabalaga, P., y Valls Llobet, C. (2005). Mujeres médicas: de la incorporación a la discriminación. Medicina Clínica (Barcelona), vol. 125 (3), 103-107.

Bracho-Riquelme, R. L.; Pescador Salas, N., y Reyes-Romero, M. (1997). Repercusión bibliométrica de adoptar el inglés como idioma único de publicación. Revista de Investigación Clínica, vol. 49 (5), 369-372.

Bruguera, M. (2001). ¿Está en crisis la investigación clínica en los hospitales españoles? Medicina Clinica (Barcelona), vol. 117 (16), 625-626.

Callahan, M.; Wears, R. L., y Weber, E. (2002). Journal prestige, publication bias, and other characteristics associated with citation of published studies in peer-reviewed journals. Journal of the American Medical Association, vol. 287 (1), 2847-2850.

De Filippo, D.; Morillo, F., y Fernández, M. T. (2008). Indicadores de colaboración científica del CSIC con Latinoamérica en bases de datos internacionales. Revista Española de Documentación Científica, vol. 31 (1), 64-84.

Dones Tacero, M.; Pérez García, J. , y Pulido San Román, A. (2008). El impacto macroeconómico de la biotecnología. Medicina Clínica (Barcelona), vol. 131 (suplemento 5), 60-65. 
Escudero Gómez, C.; Estrada Lorenzo, J. M., y Lázaro y de Mercado, P. (2008). El impacto de la investigación en la práctica clínica. Medicina Clínica (Barcelona), vol. 131 (suplemento 5), 25-29.

European Commission (2006). She Figures 2006: women and Science statistics and indicators. Luxemburgo; Office for Official Publications of the European Communities.

Frank, E. (1994). Authors criteria for selecting journals. Journal of the American Medical Association, vol. 272 (2), 163-164.

Fundación Española para la Ciencia y la Tecnología (2005). Informes sobre investigación biomédica. Madrid; Fundación Española para la Ciencia y la Tecnología.

Gingras, Y. (1984). La valeur d’une langue dans un champ scientifique. Recherches sociographiques, vol. 25 (2), 285-296.

González Alcaide, G.; Valderrama Zurián, J. C.; Navarro Molina, C.; Alonso Arroyo, A.; Bolaños Pizarro, M., y Aleixandre Benavent, R. (2007). Análisis de género de la producción científica española sobre drogodependencias en biomedicina 1999-2004. Adicciones, vol. 19 (1), 45-50.

González Alcaide, G.; Castellano Gómez, M.; Valderrama Zurián, R., y Aleixandre Benavent, R. (2008). Literatura científica de autores españoles sobre análisis de citas y Factor de Impacto en Biomedicina (1981-2005). Revista Española de Documentación Científica, vol. 31 (3), 344-365.

González Alcaide, G.; Arroyo Alonso, A., Valderrama Zurián, J. C., y Aleixandre Benavent, R. (2009a). Mujeres en la Investigación Cardiológica Española. Revista Española de Cardiología, vol. 62 (8), 945-946.

González Alcaide, G.; Bolaños Pizarro, M.; Villanueva Serrano, F. J.; Ruiz Ros, V.; González de Dios, J.; Granda Orive, J. I. de; Valderrama Zurián, J. C., y Aleixandre Benavent, R. (2009b). Dinámicas de citación y flujos de conocimiento interdisciplinar de la Biomedicina española. Actas del IX Congreso ISKO-España. Valencia 11, 12 y 13 de marzo de 2009, pp. 856-874. Valencia: Universidad Politécnica de Valencia.

González de Dios, J.; Sempere, A. P., y Aleixandre Benavent, R. (2007a). Las publicaciones biomédicas en España a debate (I): estado de las revistas neurológicas. Revista de Neurología, vol. 44 (1), 32-42.

González de Dios, J.; Sempere, A. P., y Aleixandre Benavent, R. (2007b). Las publicaciones biomédicas en España a debate (II): las 'revoluciones' pendientes y su aplicación a las revistas neurológicas. Revista de Neurología, vol. 44 (2), 101-112.

Granda Orive, J. I. de; García Río, F.; Aleixandre Benavent, R.; Valderrama Zurián, J. C.; Jiménez Ruiz, C. A.; Solano Reina, S.; Villanueva Serrano, S., y Alonso Arroyo, A. (2007). Producción española en tabaquismo a través del Science Citation Index (19992003). Situación en el contexto mundial y de la Unión Europea. Archivos de Bronconeumologia, vol. 43 (4), 212-218.

Jiménez Contreras, E. (1992). Las revistas científicas: el centro y la periferia. Revista Española de Documentación Científica, vol. 15 (2), 174-182.

Matías-Guiu, J., y García Ramos, R. (2008). El factor de impacto y las decisiones editoriales. Neurologia, vol. 23 (6), 342-348.

Medina Casaubón, J. M.; Fernández Guerrero, I. M.; Gil Montoya, J. A., y Fernández Cano, A. (2008). La investigación odontológica española en la base Science Citation Index: 
un estudio cienciométrico (1974-2006). Revista Española de Documentación Científica, vol. 31 (2), 169-189.

Olmeda Gómez, C.; Ovalle Perandones, M. A.; Perianes Rodríguez, A., y Moya Anegón, F. de (2008). Impacto internacional de la investigación y la colaboración científica de las universidades de Cataluña. 2000-2004. Revista Española de Documentación Científica, vol. 31 (4), 591-611.

Pérez Sedeño, E., y Miranda Suárez, M. J. (2008). Percepción social de la biomedicina en España. Medicina Clínica (Barcelona), vol. 131 (suplemento 5), 6-11.

Pesquero Franco, E., y Muñoz-Alonso López, G. (1997). Consideraciones teóricas y evolución del Plan Nacional de la Investigación Científica y el Desarrollo Tecnológico. Revista General de Información y Documentación, vol. 7 (1), 169-185.

Praga Terente, M. (2008). ¿Se está apoyando la investigación clínica independiente en España? Nefrología, vol. 28 (6), 575-582.

Rodés, J., y Trilla, A. (1999). Fórmulas para la integración de la formación clínica y básica en medicina. Medicina Clínica (Barcelona), vol. 11 (3), 379-382.

Rousseau, R. (2000). Are multi-authored articles cited more than single-authored ones? Are collaborations with authors from other countries more cited than collaborations within the country? A case study. Proceedings of the second Berlin workshop on Scientometrics and Informetrics. Collaboration in Science and Technology. Berlín: Gesellschaft für Wissenschaftsforschung, 173-176.

Sánchez Capelo, A. (2003). La figura del investigador sanitario. Apuntes de Ciencia y Tecnología, vol. 8.

Soriguer Escofet, F. J. C. (2009). El fracaso de la investigación clínica en España. Medicina Clínica (Barcelona), vol. 132 (6), 219-221. 